OPEN ACCESS

Edited by:

Bente Klarlund Pedersen,

Rigshospitalet, Denmark

Reviewed by:

Pradeep Kumar,

University of the Witwatersrand,

South Africa

Maha Hammad,

Dasman Diabetes Institute, Kuwait

${ }^{*}$ Correspondence:

Ozioma C. Okonkwo

ozioma@medicine.wisc.edu

Henriette van Praag

hvanpraag@health.fau.edu

${ }^{\dagger}$ These authors have contributed equally to this work

Specialty section:

This article was submitted to

Clinical Diabetes,

a section of the journal

Frontiers in Endocrinology

Received: 28 January 2021

Accepted: 30 April 2021

Published: 20 May 2021

Citation:

Gaitán JM, Moon HY,

Stremlau M, Dubal DB, Cook DB, Okonkwo OC and van Praag H (2021) Effects of Aerobic Exercise Training on Systemic Biomarkers and Cognition in Late Middle-Aged Adults at Risk for Alzheimer's Disease.

Front. Endocrinol. 12:660181. doi: 10.3389/fendo.2021.660181

\section{Effects of Aerobic Exercise Training on Systemic Biomarkers and Cognition in Late Middle-Aged Adults at Risk for Alzheimer's Disease}

\author{
Julian M. Gaitán ${ }^{1 \dagger}$, Hyo Youl Moon ${ }^{2,3,4,5+}$, Matthew Stremlau ${ }^{2}$, Dena B. Dubal ${ }^{6}$, \\ Dane B. Cook ${ }^{7,8}$, Ozioma C. Okonkwo ${ }^{1,9,10^{*}}$ and Henriette van Praag ${ }^{2,11^{*}}$
}

\begin{abstract}
${ }^{1}$ Wisconsin Alzheimer's Disease Research Center and Department of Medicine, University of Wisconsin School of Medicine and Public Health, Madison, WI, United States, ${ }^{2}$ Lab of Neurosciences, National Institute on Aging (NIA), Baltimore, MD, United States, ${ }^{3}$ Department of Education, Seoul National University, Seoul, South Korea, ${ }^{4}$ Institute of Sport Science, Seoul National University, Seoul, South Korea, ${ }^{5}$ Institute on Aging, Seoul National University, Seoul, South Korea, ${ }^{6}$ Department of Neurology and Weill Institute for Neurosciences, University of California, San Francisco, San Francisco, CA, United States, ${ }^{7}$ Department of Kinesiology, University of Wisconsin School of Education, Madison, WI, United States, ${ }^{8}$ Research Service, William S. Middleton Memorial Veterans Hospital, Madison, WI, United States, ${ }^{9}$ Geriatric Research Education and Clinical Center, William S. Middleton Memorial Veterans Hospital, Madison, WI, United States, 10 Wisconsin Alzheimer's Institute, University of Wisconsin School of Medicine and Public Health, Madison, WI, United States, ${ }^{11}$ Brain Institute and Charles E. Schmidt College of Medicine, Florida Atlantic University, Jupiter, FL, United States
\end{abstract}

Increasing evidence indicates that physical activity and exercise training may delay or prevent the onset of Alzheimer's disease (AD). However, systemic biomarkers that can measure exercise effects on brain function and that link to relevant metabolic responses are lacking. To begin to address this issue, we utilized blood samples of 23 asymptomatic late middle-aged adults, with familial and genetic risk for AD (mean age 65 years old, 50\% female) who underwent 26 weeks of supervised treadmill training. Systemic biomarkers implicated in learning and memory, including the myokine Cathepsin B (CTSB), brainderived neurotrophic factor (BDNF), and klotho, as well as metabolomics were evaluated. Here we show that aerobic exercise training increases plasma CTSB and that changes in CTSB, but not BDNF or klotho, correlate with cognitive performance. BDNF levels decreased with exercise training. Klotho levels were unchanged by training, but closely associated with change in $\mathrm{VO}_{2}$ peak. Metabolomic analysis revealed increased levels of polyunsaturated free fatty acids (PUFAs), reductions in ceramides, sphingo- and phospholipids, as well as changes in gut microbiome metabolites and redox homeostasis, with exercise. Multiple metabolites ( 30\%) correlated with changes in BDNF, but not CSTB or klotho. The positive association between CTSB and cognition, and the modulation of lipid metabolites implicated in dementia, support the beneficial effects of exercise training on brain function. Overall, our analyses indicate metabolic regulation of exercise-induced plasma BDNF changes and provide evidence that CTSB is a marker of cognitive changes in late middle-aged adults at risk for dementia.

Keywords: exercise, BDNF, metabolomics, cognition, human, Alzheimer's disease, Cathepsin B, klotho 


\section{INTRODUCTION}

Alzheimer's disease (AD) is the most common neurodegenerative disease. The accumulation of amyloid plaques and neurofibrillary tangles result in a progressive loss of brain function that inevitably leads to mental and physical disability (1). The disease has genetic components, and individuals with a family member affected by $\mathrm{AD}$ or who carry the APOE $\varepsilon 4$ allele are at increased risk (2). To date there are no effective treatment options for $\mathrm{AD}$ patients, and recent pharmacological trials have resulted in failure (3). Therefore, lifestyle interventions such as exercise training that may delay the onset of neurodegenerative conditions have become increasingly imperative $(4,5)$. In rodents, running training enhances adult hippocampal neurogenesis, synaptic plasticity, and neurotrophin levels throughout the lifespan (4). In aging humans, aerobic exercise training increases gray and white matter volume, enhances blood flow, and improves memory function (5, 6). However, human studies often utilize expensive and lowthroughput brain imaging analyses $(7,8)$ that are not practical for large population-wide studies. Systemic biomarkers that can measure the effect of exercise interventions on $\mathrm{AD}$-related outcomes quickly and at low-cost could be used to inform disease progression and for the development of novel therapeutic targets.

Several exercise biomarkers have been proposed, including growth factor BDNF, myokine CTSB, and klotho. BDNF is an apparent candidate given that this protein is upregulated in the rodent hippocampus and cortex by running and is important for adult neurogenesis, synaptic plasticity, and memory function (9-11). However, the effects of exercise training on human peripheral BDNF levels have been equivocal $(5,12-17)$. A more recent candidate marker is the myokine (18) CTSB, a lysosomal cysteine protease. Adults with cognitive impairment have lower CTSB levels in serum (19) and brain (20). Several studies indicate CTSB decreases $\beta$-amyloid $(21,22)$, whereas others report that CTSB inhibitors may reduce $\beta$-amyloid $(23,24)$. In young adults, four months of aerobic exercise training elevated plasma CTSB in association with improved fitness and memory function (25). Klotho is another circulating protein that can enhance cognition and synaptic function (26). Klotho is associated with resilience to neurodegenerative disease, possibly by supporting brain structures responsible for memory and learning (27-29). Emerging evidence suggests that its expression is upregulated by exercise $(30,31)$. Finally, peripheral metabolomic changes associated with dementia have been reported (3234 ), but have not been examined in conjunction with an exercise intervention.

In the present study we aimed to determine whether metabolomic profiles related to brain health are beneficially altered following 26 weeks of aerobic exercise training in late middle-aged adults at risk for $\mathrm{AD}$ and how such alterations relate to systemic biomarkers such as BDNF, CTSB, and klotho. Therefore, we tested the hypotheses that circulating BDNF, CTSB, and klotho would increase following exercise training and correlate with cognition and metabolomic markers of brain health.

\section{MATERIALS AND METHODS}

\section{Characteristics of Study Participants}

Participant characteristics have been published previously (35, 36). Briefly, the study enrolled 25 middle-aged adults from the Wisconsin Registry for Alzheimer's Prevention (WRAP) or the Investigating Memory in People At risk, Causes and Treatments cohort of the Wisconsin Alzheimer's Disease Research Center (WADRC IMPACT), and 23 completed the trial (see Results below). These cohorts are enriched for risk for AD by family history and the APOE $\varepsilon 4$ allele. Participants were eligible if they had a family history of $\mathrm{AD}$, were physically inactive [i.e., engaged in less than 150 minutes per week of physical activity (PA)], between 45 - 80 years of age, and cognitively healthy.

\section{Randomization and Intervention}

Details of randomization and study groups have been published elsewhere $(35,36)$. Briefly, participants were randomized after baseline assessment to either Usual Physical Activity (UPA) or Enhanced Physical Activity (EPA) using 1:1 block randomization accounting for age and sex. The UPA group was given educational material about maintaining a healthy and active lifestyle, with the presumption that they would simply maintain their usual level of PA, i.e., less than 150 minutes per week, during the 26-week study. The EPA group engaged in a supervised, progressive, moderateto-vigorous intensity aerobic exercise training program three times per week such that from week seven through 26, participants would meet or exceed public health recommendations for PA.

\section{Cardiorespiratory Fitness Assessment}

Details on the cardiorespiratory fitness $\left(\mathrm{VO}_{2}\right.$ peak) assessment have been published in detail $(35,36)$. Briefly, participants completed a graded peak exercise test on a treadmill at a constant self-selected walking speed. The treadmill incline was increased by $2.5 \%$ every two minutes until volitional exhaustion. Oxygen consumption, carbon dioxide production, and minute ventilation were recorded using a metabolic cart (TrueOne 2400, Parvomedics, Sandy, UT, USA) after standard calibration procedures to assess $\mathrm{VO}_{2}$ peak. Criteria for having achieved a $\mathrm{VO}_{2}$ peak included satisfying at least two of the following: respiratory exchange ratio $\geq 1.10$, achievement of $90 \%$ of agepredicted maximum heart rate, rating of perceived exertion $\geq 1$, and change in $\mathrm{VO}_{2}<200 \mathrm{~mL}$ with an increase in treadmill incline (37).

\section{Free-Living Physical Activity Assessment}

Details on the assessment of free-living physical activity via accelerometry have been published in detail $(35,36)$. Briefly, participants were fitted with a triaxial accelerometer (GT3X+, Actigraph, Pensacola, FL, USA) that was worn for seven consecutive days during all waking hours except during exposure to water (e.g., bathing). Data inclusion required at least 10 hours of valid wear time per day for a minimum of three weekdays and one weekend day, and data were processed as previously described (36) to yield counts of time spent sedentary, or spent in light, moderate, or vigorous PA. 


\section{Cognitive Function Test}

Details of the cognitive function testing have been published previously $(35,36)$. A trained rater administered a cognitive test battery to each participant. The Mini-Mental State Examination (MMSE) (38) was administered to assess global cognitive function and confirm participants' cognitively unimpaired status. The California Verbal Learning Test-II (CVLT) (39) was used to assess episodic verbal memory and consists of five learning trials (resulting in the CVLT Total score) and a 20-minute post-learning recall trial (the CVLT Long Delay score). The Delis-Kaplan Executive Function System (D-KEFS) (40) was used to assess executive function. It is comprised of Verbal Fluency, Design Fluency, Trail Making, Sorting, and Color-Word Interference (CWI) subtests. As with our prior publication (36), only the CWI subtest was analyzed for the present report due to its particular sensitivity to executive dysfunction in pre-clinical $\mathrm{AD}$ (41). Although the cognitive outcomes analyzed in this study were objective scores (i.e., word counts for CVLT Total and Long Delay scores and time to completion for D-KEFS CWI), the technician responsible for administering cognitive testing was unblinded to treatment allocation.

\section{Blood Sample Collection}

Blood samples were collected after a 12-hour fast from food, caffeine, and alcohol. Samples for plasma analyses were collected into a $10 \mathrm{~mL}$ EDTA vacutainer which was then gently inverted 10-12 times. Within one hour of collection, EDTA tubes were centrifuged at $3000 \mathrm{rpm}$ for 15 minutes at room temperature. Cell-free plasma was then aliquoted into cryovials and stored at $80^{\circ} \mathrm{C}$ until assayed for CTSB, BDNF, and metabolites. Blood samples for serum analyses were collected into a $9 \mathrm{~mL}$ vacutainer containing no anticoagulant or additive. The samples were allowed to clot for no more than 30 minutes and were then centrifuged at $3000 \mathrm{rpm}$ for 10 minutes at $4^{\circ} \mathrm{C}$. Serum was then aliquoted into cryovials and stored at $-80^{\circ} \mathrm{C}$ and was not thawed until assays were run. An additional $4 \mathrm{~mL}$ whole blood were collected into a $4 \mathrm{~mL}$ vacutainer containing no additive and was sent to a clinical laboratory to be analyzed for triglycerides, low density lipoprotein (LDL), high density lipoprotein (HDL), and non-HDL cholesterol levels.

\section{BDNF, CTSB, and Klotho Quantification by Enzyme-Linked Immunosorbent Assay (ELISA)}

BDNF and CTSB quantification in plasma was performed using total BDNF ELISA (R\&D Systems, Minneapolis, MN, USA) and CTSB ELISA (Abcam, Cambridge, MA, USA) kits, in accordance with the manufacturers' specifications. Frozen plasma samples were thawed at room temperature $\left(25^{\circ} \mathrm{C}\right)$ and held on ice. Samples were loaded in duplicate on a microplate and incubated in accordance with the manufacturers' protocols. Each assay included recombinant protein as a positive control. Plates were read using SpectraMax Plus 384 Microplate Reader (Molecular Devices, Sunnyvale, CA, USA).

Soluble $\alpha$-klotho was measured using a solid-phase sandwich ELISA (Immuno-Biological Laboratories, Takasaki, Japan) (42) as described $(26,43)$. Briefly, serum was diluted four-fold with the immunoassay buffer, a standard curve was created by serial dilution of recombinant human $\alpha$-klotho protein, and diluted serum was loaded in duplicate onto a plate pre-coated with affinity-purified anti-human klotho (67G3) mouse IgG monoclonal antibody. Control samples were included as references for each plate to enable accurate interplate comparisons. Following incubation for $1 \mathrm{~h}$ at room temperature, the plates were washed five times with washing buffer, horseradish peroxidase-conjugated anti-human klotho $(100 \mu \mathrm{l}, 91 \mathrm{~F} 1)$ mouse IgG monoclonal antibody was added, plates were incubated for $30 \mathrm{~min}$ at room temperature, and then plates were washed five times. The reaction was visualized by addition of $100 \mu \mathrm{l}$ of chromogenic substrate for $30 \mathrm{~min}$ at room temperature and then stopped with $100 \mu \mathrm{l}$ of $1 n \mathrm{H}_{2} \mathrm{SO}_{4}$ The absorbance at $450 \mathrm{~nm}$ was measured on a Spectramax 190 plate reader (Molecular Devices, Sunnyvale, CA, USA), and $\alpha$ klotho levels were calculated using the SoftMax Pro software (Molecular Devices). Samples with coefficient of variation (CV) above $10 \%$ were re-run. Final assay values had an average CV of $2.3 \%$ for serum.

\section{Metabolomics Analysis}

\section{Sample Preparation for Metabolomic Analysis}

Samples were prepared using the automated MicroLab STAR ${ }^{\circledR}$ system (Hamilton Company, Reno, NV, USA). Several recovery standards were added prior to the first step in the extraction process for quality control purposes. Proteins were extracted by precipitation in methanol under vigorous shaking for 2 minutes (Glen Mills GenoGrinder 2000), followed by centrifugation. The resulting extract was divided into five fractions: two for analysis by two separate reverse phase ultrahigh performance liquid chromatography-tandem mass spectroscopy (RP-UPLC-MS/ MS) methods with positive ion-mode electrospray ionization (ESI), one for analysis by RP/UPLC-MS/MS with negative ionmode ESI, one for analysis by hydrophilic interaction chromatography (HILIC)/UPLC-MS/MS with negative ionmode ESI, and one for potential future analyses. Samples were placed briefly on a TurboVap ${ }^{\circledR}$ (Zymark, USA) to remove organic solvent. Sample extracts were stored overnight under nitrogen before analysis.

\section{UPLC-MS/MS}

All methods utilized an ACQUITY UPLC apparatus (Waters, Milford, MA, USA) and a Q-Exactive high resolution/accurate mass spectrometer (Thermo Scientific, Waltham, MA, USA) interfaced with a heated ESI source and Orbitrap mass analyzer operated at 35,000 mass resolution. Further details of this analysis are in the Supplementary Information.

\section{Statistics}

Data were analyzed using SPSS 26.0 (IBM Corp., Armonk, NY). Independent samples t-tests (for continuous variables) and Fischer's exact tests (for categorical variables) were used to analyze baseline differences between treatment groups. Raw metabolite values were normalized in terms of raw area counts by $\log$ transformation and rescaled to set the median equal to 1 . 
Missing values, if any, were imputed with the minimum observed value for each metabolite. Two-way repeated measures ANOVA was conducted to test for differences in metabolite levels from baseline to post-intervention by group. Paired samples t-tests were conducted to test differences in metabolites and CTSB, BDNF, and klotho from baseline to post-intervention within each group. Pearson correlations were conducted among systemic biomarkers and cognitive, fitness, physical activity, laboratory, and metabolomic measures.

\section{RESULTS}

\section{Study Group Participant Properties and Timeline of the Experiments}

Twenty-five participants enrolled in the study. One participant was excluded after baseline assessment due to an adverse finding on ultrasound imaging, and one discontinued participation in EPA due to an unexpected surgery, resulting in 23 participants completing the study. Participants had a mean age of 64.9 years at baseline. Approximately 50\% of the participants were female. On average, participants had 16 years of education, all had parental history of $\mathrm{AD}, 35 \%$ carried at least one copy of the APOE $\varepsilon 4$ allele, and all were cognitively healthy as further indicated by their baseline MMSE score (mean $=29.61 \pm 0.58)$. There were no significant differences on these baseline characteristics between the groups (Table 1). A timeline of the blood sample collection, cognitive testing, and duration of the intervention is depicted in Figure 1A.

\section{Changes in Systemic Biomarkers}

Post-intervention CTSB, BDNF, and klotho values in the UPA group were not significantly different compared to baseline levels (CTSB: $t_{(11)}=1.78, p=0.102$; BDNF: $t_{(11)}=-0.88, p=0.396$; klotho: $t_{(11)}=1.32, p=0.215$; Figures 1B-D). The EPA group displayed a significant increase in plasma CTSB $\left(t_{(10)}=3.235, p=0.009\right)$, reduced $\operatorname{BDNF}\left(t_{(10)}=-3.90, p=0.003\right)$, and unchanged klotho levels $\left(t_{(10)}=-0.09, p=0.927\right)$ after the exercise intervention (Figures 1B-D).

\section{Cardiorespiratory Fitness, Physical Activity, and Cognition Following Exercise Training}

Effects of the 26-week exercise training intervention on $\mathrm{VO}_{2}$ peak, physical activity, sedentariness, and cognition have been

TABLE 1 | Baseline characteristics of the study sample.

\begin{tabular}{lccc}
\hline & UPA $(\mathbf{n}=\mathbf{1 2})$ & EPA $(\mathbf{n = 1 1 )}$ & $\boldsymbol{p}$ \\
\hline Age & $63.92(5.19)$ & $65.88(4.00)$ & 0.33 \\
Female sex, $\mathbf{n}$ & 6 & 5 & 0.99 \\
Education & $16.67(2.57)$ & $16.27(2.33)$ & 0.71 \\
Parental history of AD, $\mathbf{n}$ & 12 & 11 & - \\
APOE \&4 positive, $\mathbf{n}$ & 5 & 3 & 0.67 \\
\hline
\end{tabular}

Data are mean (standard deviation). Usual Physical Activity (UPA); Enhanced Physical Activity (EPA); Alzheimer's disease (AD); apolipoprotein E epsilon 4 (APOE ع4). published previously (36). Briefly, the EPA group exhibited a greater increase in $\mathrm{VO}_{2}$ peak and moderate to vigorous physical activity (MVPA), decrease in sedentary behavior, and improvement on the D-KEFS CWI than the UPA group. The improvement on the D-KEFS CWI was correlated with $\mathrm{VO}_{2}$ peak (36).

\section{BDNF, CTSB, and Klotho Levels and Cognition}

Change in plasma CTSB levels correlated significantly with change in the CVLT Total score (Figure 2A, $\mathrm{r}=.44, p<.05$ ) and exhibited a weak but non-significant correlation with the DKEFS CWI (Figure 2B, $\mathrm{r}=.31, p=.16$ ), but not with CVLT Long Delay (Figure S1A), $\mathrm{VO}_{2}$ peak (Figure 2C), MVPA, or sedentary behavior (Table 2). Change in plasma BDNF was not significantly correlated with any of the cognitive test scores (all r's <.22, $p$ 's >.32; Figures 2D, E and S1B), $\mathrm{VO}_{2}$ peak (Figure 2F), MVPA, or sedentary behavior (Table 2). Change in klotho level was significantly correlated with change in $\mathrm{VO}_{2}$ peak (Figure 2I; $\mathrm{r}=0.562, p=.005$; Table 2) and exhibited a weak but nonsignificant correlation with MVPA $(\mathrm{r}=.33, p=.12$; Table 2) but not with cognitive test scores (Figures 2G, $\mathbf{H}$ and S1C).

\section{Circulating Lipids}

Lipid panels were unchanged. There were no significant associations between CTSB, BDNF, or klotho, and blood lipids (all r's <.36, all p's >.09; Table 3).

\section{Metabolomic Profiling}

For this study, 46 total plasma samples collected at baseline and after 26 weeks of UPA or EPA (post-intervention) were sent to Metabolon for metabolomics analysis (Figure 1A). A total of 881 named biochemicals were identified, with lipid metabolites being the most prevalent (Figure 1E). Of the 881 total metabolites (Table S1), statistical analyses showed that there was a significant modulation of 78 metabolites $(p<0.05)$ in the UPA condition, while in the EPA group there was a significant change in 115 metabolites $(p<0.05)$ from baseline to post-intervention. Changes in lipid metabolites accounted for $\sim 50 \%$ of total significantly modulated metabolites in EPA $[57(p<0.05)]$ and $~ 35 \%$ (nonoverlapping) in UPA [28 $(p<0.05)]$. We focus on 40 lipid metabolites clustered in pathways implicated in $\mathrm{AD}$ and exercise (Table 4), as well as on 15 metabolites relevant to oxidative stress and the gut microbiome in the EPA group (Table 5). Of those 55 metabolites, there was 1 significant correlation with $\mathrm{VO}_{2}$ peak, 5 with MVPA, 19 with BDNF, 1 with CTSB, and none with klotho (Table 4; Table 5 and Table S2). The entire data set is available upon request and has been deposited in the Metabolomics Workbench, Project ID PR001121 (http://doi.org/10.21228/M8SX23).

\section{Changes in Sphingolipid Metabolism}

Sphingolipids are multifunctional lipids, composed of a ceramide (a lipid composed of sphingosine and a fatty acid) and a polar head group (e.g. phosphocholine), that can regulate cell structure (sphingomyelins) and signaling (ceramides, sphingosine-1-phosphate) $(44,45)$. In the bloodstream, sphingolipids are transported by lipoproteins, primarily by LDL (46). Reductions in several ceramides [e.g., ceramide (d18:1/ 
A

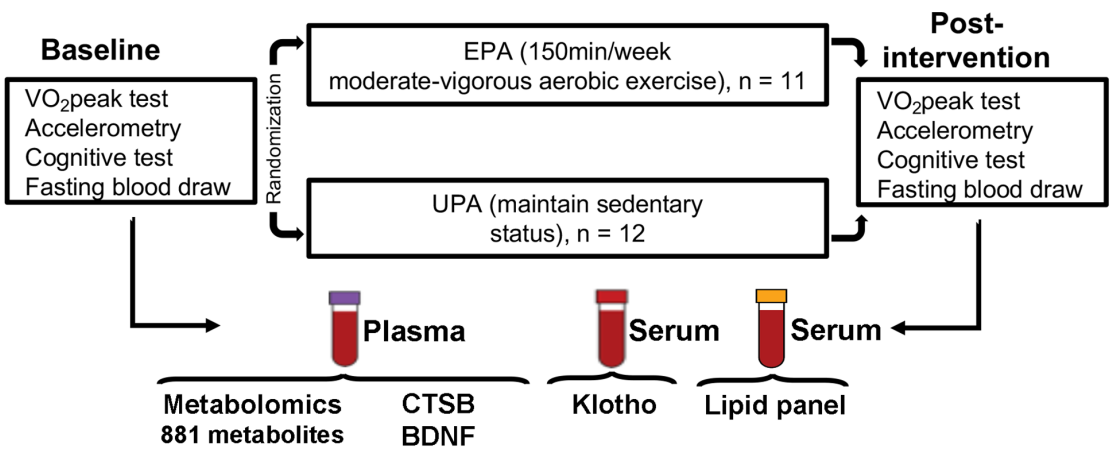

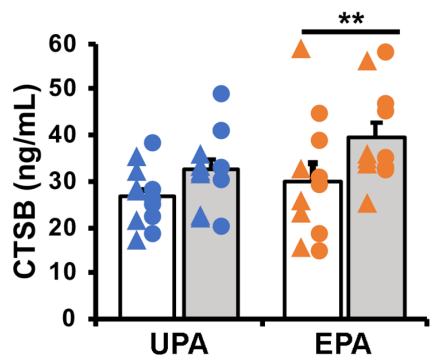

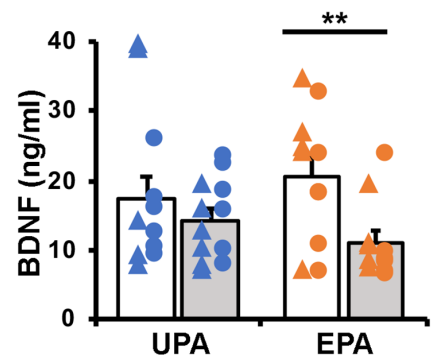

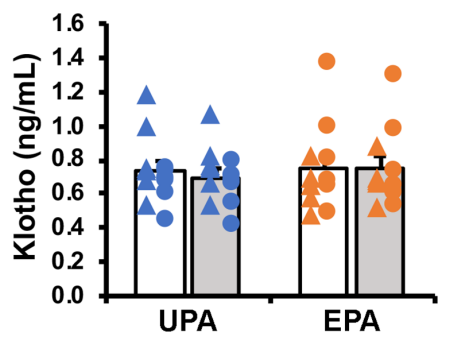

E

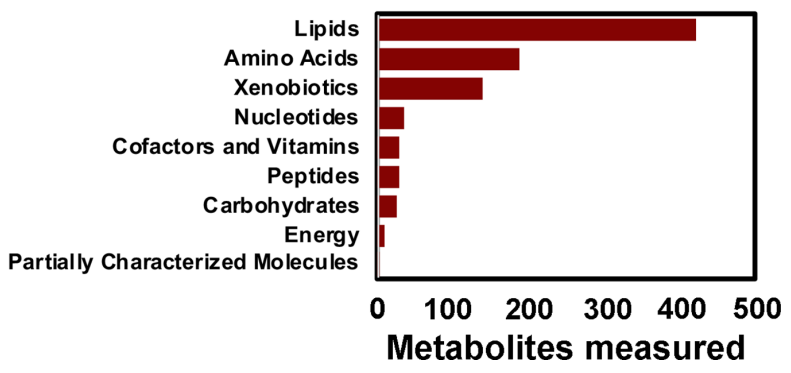

FIGURE 1 | Study design and effects of exercise on circulating biomarkers. (A) Overview of study design, exercise training intervention [Usual Physical activity (UPA) or Enhanced Physical Activity (EPA)], and blood sample analyses. The list of outcomes is specific to the present report; other outcomes were measured as part of the trial and are described elsewhere (35). (B-D) Effects of the 26-week aerobic exercise intervention on systemic biomarkers. (B) Plasma CTSB increased in the EPA group post-intervention as compared to baseline. (C) Plasma BNDF decreased in the EPA group. (D) Serum klotho levels did not change with exercise. (E) Plasma-derived metabolites by super pathway. ${ }^{* *} p<0.01$ Cathepsin B (CTSB); brain-derived neurotrophic factor (BDNF); Usual Physical Activity (UPA); Enhanced Physical Activity (EPA).

14:0, d16:1/16:0) and ceramide (d18:1/20:0, d16:1/22:0, d20:1/18:0) and $\mathrm{N}$-palmitoyl-sphinganine (d18:0/16:0)] were observed in EPA postintervention, as compared to baseline. In addition, dihydrosphingomyelins [sphingomyelin (d18:0/20:0, d16:0/22:0), palmitoyl dihydrosphingomyelin (d18:0/16:0), behenoyl dihydrosphingomyelin (d18:0/22:0)], sphingosines [sphingosine, sphingosine 1-phosphate], and sphingolipid synthesis [sphinganine, sphinganine 1-phosphate, sphingadienine] decreased. Change in sphinganine, sphinganine-1-phosphate, and sphingosine was tightly correlated with plasma BDNF change, (Figure 3 and Table 4) but not with klotho or CTSB or measures of cognition or $\mathrm{VO}_{2}$ peak (Table S2).

Alterations in Phospholipid and Fatty Acid Metabolism Phospholipids are key constituents of the plasma membrane. Phospholipids have a hydrophobic tail consisting of two fatty acids, and a hydrophilic head (e.g., choline, ethanolamine, serine or inositol) to form phosphatidylcholine (PC), -ethanolamine (PE), -serine (PS), or -inositol (PI). Hydrolysis of phospholipids by phospholipase A2 generates free fatty acids (e.g. arachidonate), 


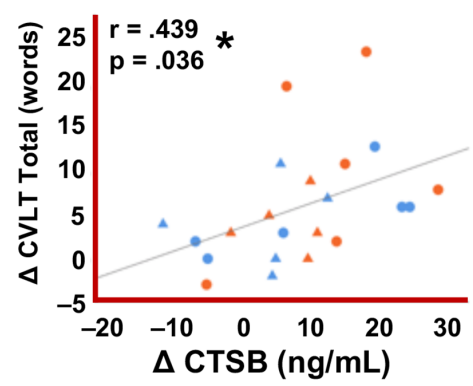

B

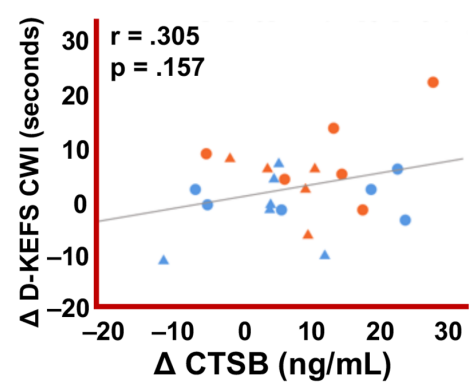

c

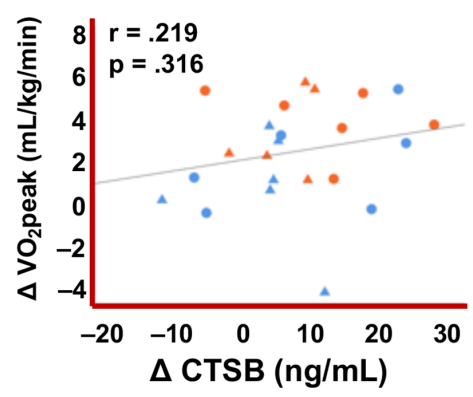

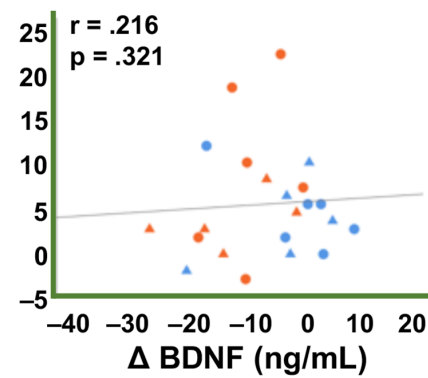

E

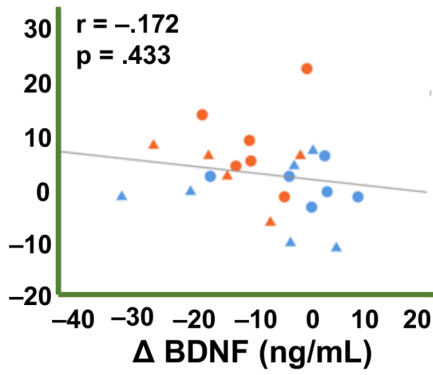

$\mathbf{F}$

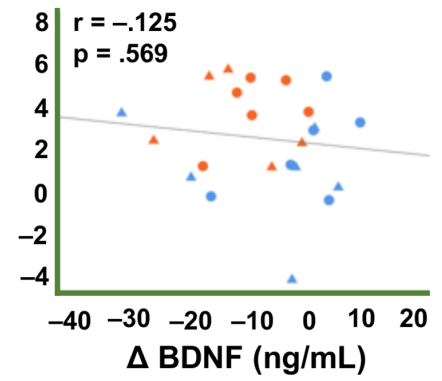

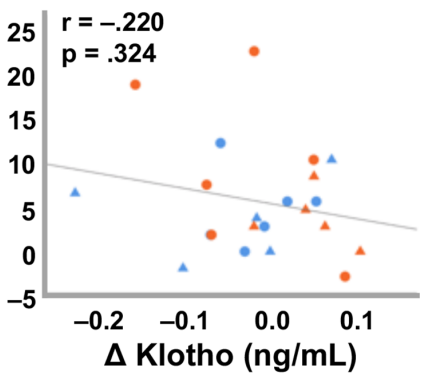

H

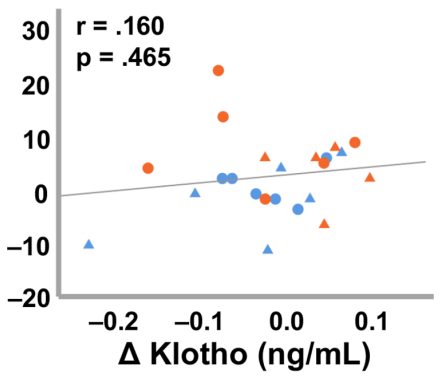

I

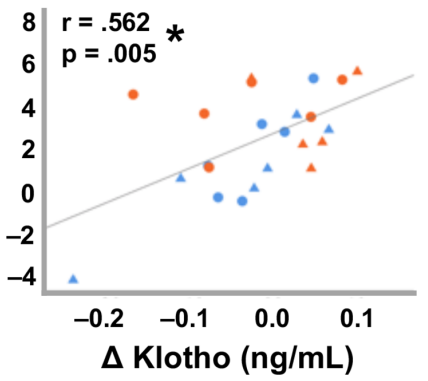

FIGURE 2 | Correlations between changes in systemic biomarkers with cognitive and cardiorespiratory fitness measures. (A) Change in CTSB was significantly correlated with verbal learning and memory assessed by the CVLT Total recall score. (B) Change in CTSB was correlated, albeit not significantly, with executive function on the D-KEFS CWI. (C) There was no correlation between CTSB change and cardiorespiratory fitness. (D-F) Change in BDNF was not significantly correlated with cognitive performance or cardiorespiratory fitness. (G, H) Change in klotho was not significantly correlated with cognitive performance. (I) Change in klotho was significantly correlated with cardiorespiratory fitness. A decrease in the raw D-KEFS CWI scores indicates improvement on this outcome, so D-KEFS CWI scores were inverted such that a positive change indicates improvement in this figure. ${ }^{*} p<.05$. Usual Physical Activity (UPA); Enhanced Physical Activity (EPA); cathepsin B (CTSB); brain-derived neurotrophic factor (BDNF); cardiorespiratory fitness $\left(\mathrm{VO}_{2}\right.$ peak); California Verbal Learning Test (CVLT); Delis-Kaplan Executive Function System Color-Word Interference (D-KEFS CWI).

and 1-lysolipids which are ligands for G-protein coupled receptors that influence development, physiology and disease $(47,48)$. Post-intervention, the EPA group exhibited notable changes in phospholipid metabolism. Lysophospholipids (7 out of 26 metabolites) were elevated and one metabolite, 1-stearoylglycerophophatidylserine (1-stearoyl GPS, 18:0), decreased. Reductions were also observed in one phosphatidylcholine (PC), multiple phosphatidylethanolamines (PE) (9 out of 12 metabolites), and two phosphatidylinositols (PI). In addition, choline increased and choline phosphate (phosphocholine), phosphoethanolamine, and glycerophosphoethanolamine decreased. Changes in 1-stearoyl-GPS (18:0), 1-palmitoyl-2arachidonoyl-GPI (16:0/20:4), 1-palmitoyl-2-arachidonoyl-GPE (16:0/20:4) 1-stearoyl-2-arachidonoyl-GPE (18:0/20:4), 1-oleoyl2-arachidonoyl-GPE (18:1/20:4), 1-palmitoyl-2-stearoyl-GPC (16:0/18:0) [also correlated with $\mathrm{VO}_{2}$ peak, (Table S2)], 
TABLE 2 | Correlations among cardiorespiratory fitness, physical activity, sedentariness and systemic biomarkers.

\begin{tabular}{|c|c|c|c|c|c|c|}
\hline & \multicolumn{2}{|c|}{$\Delta$ CTSB } & \multicolumn{2}{|c|}{$\Delta$ BDNF } & \multicolumn{2}{|c|}{$\Delta$ Klotho } \\
\hline & $r$ & $p$ & $r$ & $p$ & $r$ & $p$ \\
\hline$\Delta \mathrm{VO}_{2}$ peak (mL/kg/min) & .219 & .316 & -.125 & .569 & .562 & .005 \\
\hline$\Delta$ MVPA (min) & -.112 & .610 & -.275 & .204 & .332 & .122 \\
\hline$\Delta$ Sedentary time (min) & .035 & .875 & -.040 & .856 & -.257 & .237 \\
\hline
\end{tabular}

Cathepsin B (CTSB); brain-derived neurotrophic factor (BDNF); Moderate to vigorous physical activity (MVPA)

glycerophosphoethanolamine, phosphoethanolamine, and choline phosphate correlated closely with change in BDNF, but not with CTSB or klotho (Figure 4; Table 4 and Table S2).

Phospholipase activity is also involved in generation of free fatty acids (49). Indeed, the levels of polyunsaturated fatty acids (PUFAs) were increased in the EPA group at post-intervention as compared to baseline, including dihomo-linolenate $(20: 3 \mathrm{n} 3$ or $\mathrm{n} 6)$, arachidonate (20:4n6), docosapentaenoate (n6 DPA; 22:5n6), and docosahexaenoate (DHA; 22:6n3), while no significant changes were noted in saturated fatty acids (Table $\mathbf{4}$ and Figure S2).

\section{Perturbations in Redox Homeostasis}

Higher levels of methionine sulfoxide were detected in the EPA group at post-intervention as compared to baseline. In addition, taurine and hypotaurine levels decreased in the EPA group at post-intervention as compared to baseline. Both taurine and hypotaurine change correlated significantly with BDNF change (Figure 5 and Table 5). Furthermore, the levels of the polyamine spermidine (50) were decreased in the EPA group (postintervention versus baseline). A significant positive correlation was observed between BDNF, but not CTSB or klotho, and spermidine (Table 5 and Table S2).

\section{Differences in Molecules Originating in the Gut Microbiome}

Changes from baseline to post-intervention were observed in catabolites of phenylalanine and tyrosine generated by the microbiome. In the EPA group, levels of 3-(4-hydroxyphenyl) lactate and phenylacetylglutamine were higher, while phenylpyruvate levels were lower (Table 5). Tryptophan-derived serotonin and indole propionate (IPA) levels also declined in the EPA group at postintervention as compared to baseline. The change in serotonin was closely linked to BDNF change, whereas IPA was significantly associated with CTSB (Figure 6).
Additionally, a number of benzoate-derived compounds were altered over the course of the study. Benzoate metabolites are simple carboxylic acids produced from the microbial degradation of dietary aromatic compounds in the intestine, such as polyphenols (51), purines and aromatic organic acids. 3hydroxyhippurate sulfate and o-cresol sulfate were elevated in EPA group, whereas 3-methoxycatechol sulfate (2) was reduced at post-intervention as compared to baseline. The metabolite 3hydroxyhippurate sulfate was the only one evaluated that displayed a positive correlation with cognition (D-KEFS CWI, $\mathrm{r}=0.44, p=0.033)$.

\section{Protein Turnover}

Higher levels of hydroxyproline $(p=0.076)$ and N-methylproline $(p<0.05)$ were detected in the EPA group at post-intervention as compared to baseline. Increased levels of free methylated or hydroxylated amino acids are often indications of enhanced protein degradation, which may reflect changes in muscle composition as a result of physical activity. In particular, hydroxyproline, is specific to the collagen family of proteins (52). Also, changes were noted in aminosugars (e.g., increased glucuronate, decreased $\mathrm{N}$-acetylneuraminate) in the EPA group between postintervention and baseline. Elevated $\mathrm{N}$-acetylneuraminate has been observed to be increased in the cerebrospinal fluid of $\mathrm{AD}$ patients (53). N-acetylneuraminate change was closely correlated with BDNF (Table 5).

\section{DISCUSSION}

In absence of therapeutics for $\mathrm{AD}$, exercise has become an important lifestyle factor that may prevent or delay onset of the disease $(5,11)$. Measuring the effects of exercise on systemic biomarkers associated with risk for $\mathrm{AD}$ and relating them to key metabolomic alterations may further prevention, monitoring, and treatment efforts. We evaluated three systemic biomarkers, CTSB, BDNF, and klotho, and performed a metabolomics analysis in the same subjects in blood samples taken at baseline and after a 26-week aerobic intervention. Changes in systemic biomarkers and metabolites were generally consistent with the beneficial effects of exercise for brain function. Plasma CTSB was elevated with EPA and positively associated with cognitive function. Plasma BDNF was diminished in EPA. Serum klotho was unchanged but positively correlated with cardiorespiratory fitness. Metabolomic analysis

TABLE 3 | Lipid panel results and correlations among lipids and systemic biomarkers.

\begin{tabular}{|c|c|c|c|c|c|c|c|c|c|c|}
\hline & \multicolumn{2}{|c|}{ UPA (n=12) } & \multicolumn{2}{|c|}{ EPA $(n=11)$} & \multicolumn{2}{|c|}{$\Delta$ CTSB } & \multicolumn{2}{|c|}{$\Delta$ BDNF } & \multicolumn{2}{|c|}{$\Delta$ Klotho } \\
\hline & Baseline & Post-intervention & Baseline & Post-intervention & $r$ & $p$ & $\mathbf{r}$ & $p$ & $\mathbf{r}$ & $p$ \\
\hline Total Chol (mg/dL) & 197.50 (30.16) & 186.75 (34.66) & 206.00 (39.80) & $199.36(38.14)$ & .212 & .332 & .188 & .389 & .248 & .254 \\
\hline Triglycerides (mg/dL) & $124.75(50.29)$ & $119.17(57.35)$ & $114.73(65.89)$ & $100.64(58.44)$ & .247 & .255 & -.011 & .960 & -.048 & .829 \\
\hline HDL Chol (mg/dL) & $54.00(17.37)$ & $50.58(15.98)$ & $57.73(17.52)$ & $54.36(10.71)$ & -.255 & .241 & -.115 & .602 & .364 & .087 \\
\hline LDL Chol (mg/dL) & $118.75(27.58)$ & $112.42(28.00)$ & $125.36(34.72)$ & $124.82(34.19)$ & .276 & .202 & .264 & .223 & .130 & .555 \\
\hline Non-HDL Chol (mg/dL) & $143.50(29.76)$ & $136.17(30.72)$ & $148.27(34.87)$ & $145.00(35.97)$ & .324 & .131 & .243 & .264 & .109 & .620 \\
\hline
\end{tabular}

Data are mean (standard deviation). Usual Physical Activity (UPA); Enhanced Physical Activity (EPA); cathepsin B (CTSB); brain-derived neurotrophic factor (BDNF); Cholesterol (Chol); high density lipoprotein (HDL); low density lipoprotein ( $L D L)$. 
TABLE 4 | Correlations between BDNF and lipid metabolite changes.

\begin{tabular}{|c|c|c|c|c|c|}
\hline \multirow{3}{*}{$\begin{array}{l}\text { Pathway } \\
\text { Lysophospholipid }\end{array}$} & \multirow{3}{*}{\begin{tabular}{|l} 
Metabolite Name \\
1-arachidonoyl-GPI $(20: 4)$
\end{tabular}} & \multicolumn{2}{|c|}{$\Delta$ BDNF } & \multirow{2}{*}{\multicolumn{2}{|c|}{$\begin{array}{c}\text { UPA EPA } \\
\text { Fold of change }\end{array}$}} \\
\hline & & \multirow{2}{*}{$\begin{array}{c}\mathbf{r} \\
-.005\end{array}$} & \multirow{2}{*}{$\begin{array}{c}p \\
.983\end{array}$} & & \\
\hline & & & & 1.15 & $1.27^{*}$ \\
\hline & 1-linoleoyl-GPI (18:2) & -.404 & .056 & 1.04 & $1.37^{*}$ \\
\hline & 1-oleoyl-GPI (18:1) & -.069 & .754 & 1.14 & $1.53^{*}$ \\
\hline & 1-palmitoyl-GPI (16:0) & .001 & .998 & 1.32 & $1.37 \dagger$ \\
\hline & 1-stearoyl-GPI (18:0) & -.107 & .626 & 1.16 & $1.46^{*}$ \\
\hline & 2-palmitoyl-GPC (16:0)† & -.143 & .515 & 1.02 & $1.33^{*}$ \\
\hline & 1-linoleoyl-GPG (18:2)‡ & -.078 & .725 & 0.96 & $1.22 \dagger$ \\
\hline & 1-stearoyl-GPS (18:0)‡ & .510 & .013 & 0.79 & $.039^{*}$ \\
\hline \multirow[t]{9}{*}{ Phosphatidylethanolamine } & 1,2-dipalmitoyl-GPE (16:0/16:0)† & .151 & .492 & 0.86 & $0.58^{*}$ \\
\hline & 1-palmitoyl-2-oleoyl-GPE (16:0/18:1) & .352 & .099 & 0.95 & $0.71^{*}$ \\
\hline & 1-palmitoyl-2-linoleoyl-GPE (16:0/18:2) & -.073 & .740 & 0.99 & $0.78^{*}$ \\
\hline & 1-palmitoyl-2-arachidonoyl-GPE (16:0/20:4) & .503 & .014 & 1.08 & $0.75^{*}$ \\
\hline & 1-stearoyl-2-oleoyl-GPE (18:0/18:1) & .311 & .149 & 0.85 & $0.68^{*}$ \\
\hline & 1-stearoyl-2-linoleoyl-GPE (18:0/18:2)‡ & .059 & .790 & 0.95 & $0.78^{*}$ \\
\hline & 1-stearoyl-2-arachidonoyl-GPE (18:0/20:4) & .608 & .002 & 1.05 & $0.67^{\star}$ \\
\hline & 1-oleoyl-2-linoleoyl-GPE (18:1/18:2)ł & .102 & .645 & 0.91 & $0.81^{*}$ \\
\hline & 1-oleoyl-2-arachidonoyl-GPE (18:1/20:4)‡ & .658 & .001 & 1.10 & $0.74^{*}$ \\
\hline \multirow[t]{3}{*}{ Phosphatidylinositol } & 1-palmitoyl-2-linoleoyl-GPI (16:0/18:2) & -.107 & .628 & 0.81 & $0.76+$ \\
\hline & 1-palmitoyl-2-arachidonoyl-GPI (16:0/20:4)‡ & .028 & .898 & 0.93 & $0.69^{*}$ \\
\hline & 1-stearoyl-2-arachidonoyl-GPI (18:0/20:4) & .352 & .099 & 0.98 & $0.85^{\star}$ \\
\hline Phosphatidylcholine & 1-palmitoyl-2-stearoyl-GPC (16:0/18:0) & .592 & .003 & 1.01 & $0.83^{*}$ \\
\hline \multirow[t]{4}{*}{ Polyunsaturated Fatty Acid (n3 \& n6) } & arachidonate $(20: 4 n 6)$ & .070 & .751 & 1.14 & $1.27^{*}$ \\
\hline & docosahexaenoate (dha; 22:6n3) & -.224 & .305 & 1.12 & $1.20 \dagger$ \\
\hline & docosapentaenoate (n6 dpa; 22:5n6) & -.191 & .383 & 1.21 & $1.38^{*}$ \\
\hline & dihomo-linolenate (20:3n3 or n6) & -.074 & .736 & 1.13 & $1.23^{*}$ \\
\hline \multirow[t]{2}{*}{ Ceramides } & ceramide (d18:1/20:0,d16:1/22:0, d20:1/18:0)† & .262 & .227 & 0.93 & $0.82^{*}$ \\
\hline & ceramide (d18:1/14:0, d16:1/16:0)† & .141 & .522 & 0.91 & $0.73^{\star}$ \\
\hline \multirow[t]{3}{*}{ Phospholipid metabolism } & choline phosphate & .858 & $<.001$ & 0.87 & $0.59^{*}$ \\
\hline & glycerophosphoethanolamine & .788 & $<.001$ & 0.99 & $0.77^{*}$ \\
\hline & phosphoethanolamine & .854 & $<.001$ & 0.85 & $0.46^{*}$ \\
\hline \multirow[t]{3}{*}{ Dihydrosphingomyelins } & sphingomyelin (d18:0/20:0, d16:0/22:0) & .153 & .487 & 0.90 & $0.71^{*}$ \\
\hline & behenoyl dihydrosphingomyelin (d18:0/22:0) & .371 & .081 & $0.85 \dagger$ & $0.71^{*}$ \\
\hline & palmitoyl dihydrosphingomyelin (d18:0/16:0)‡ & .175 & .424 & 0.97 & $0.88^{*}$ \\
\hline Dihydroceramides & n-palmitoyl-sphinganine (d18:0/16:0) & .232 & .286 & 0.91 & $0.75^{\star}$ \\
\hline \multirow[t]{3}{*}{ Sphingolipid Synthesis } & sphinganine & .724 & $<.001$ & 0.65 & $0.41^{*}$ \\
\hline & sphinganine-1-phosphate & .727 & $<.001$ & 0.84 & $0.55^{\star}$ \\
\hline & sphingadienine & .711 & $<.001$ & 0.57 & $0.28^{*}$ \\
\hline \multirow[t]{3}{*}{ Sphingosines } & sphingosine & .664 & .001 & 0.58 & $0.34^{*}$ \\
\hline & sphingosine 1-phosphate & .668 & $<.001$ & 0.91 & $0.68^{*}$ \\
\hline & hexadecasphingosine $(\mathrm{d} 16: 1) \ddagger$ & .754 & $<.001$ & 0.78 & $0.44^{*}$ \\
\hline
\end{tabular}

Correlations between changes in plasma BDNF levels and lipid metabolites (bolded $p$ values indicate significance). Fold changes of the lipid metabolites in the UPA and EPA groups is listed. ${ }^{*}$ indicates a metabolite that was identified but has not been confirmed based on a standard of that metabolite. ${ }^{*} p<.05 .{ }^{\dagger} .05<p<.10$. brain-derived neurotrophic factor (BDNF); Usual Physical Activity (UPA); Enhanced Physical Activity (EPA).

revealed increased levels of PUFAs and lysophospholipids, and reductions in ceramides, sphingo- and phospho-lipids in EPA. Metabolite levels indicated oxidative stress with EPA; methionine sulfoxide increased and taurine, hypotaurine and spermidine levels decreased. Changes in multiple metabolites correlated closely with BDNF, suggesting that this neurotrophin is regulated by metabolic factors. Overall, the positive correlation between CTSB and cognition, and the substantial modulation of lipid metabolites implicated in dementia, support the beneficial effects of aerobic exercise on brain health in asymptomatic individuals at risk for AD.

Plasma CTSB is upregulated by aerobic exercise training and associated with cognition in young adults (25). In the present study we show that CTSB is increased in late-middle-aged adults after 26 weeks of aerobic training, indicating that CTSB levels change after prolonged activity $(25,54,55)$ whereas shorter term aerobic exercise protocols $(56,57)$, and resistance training $(58$,
59) show no change. The present findings in late-middle aged adults at risk for $\mathrm{AD}$ extend findings from healthy younger adults that CTSB levels were positively correlated with a hippocampusdependent visual memory task after a four-month aerobic exercise intervention (25). Our results demonstrate that verbal learning and memory, measured by the CVLT, correlated positively with CTSB but was not related to BDNF or klotho. Performance on the CVLT is known to decline in preclinical AD (60). Given that context, the present correlation between CTSB and verbal learning and memory suggests that CTSB may be useful as a marker for cognitive changes relevant to hippocampal function after exercise in a population at risk for $\mathrm{AD}$. In addition, a weak but non-significant positive association between CTSB and the D-KEFS CWI score, an executive function test considered to be mediated by the prefrontal cortex, was observed. A recent study of habitually exercising male adults 
TABLE 5 | Correlations between non-lipid metabolites and systemic biomarkers.

\begin{tabular}{|c|c|c|c|c|c|c|c|c|c|c|}
\hline \multirow[t]{2}{*}{ Super Pathway } & \multirow[t]{2}{*}{ Sub Pathway } & \multirow[t]{2}{*}{ Metabolite Name } & \multicolumn{2}{|c|}{$\Delta \mathrm{CTSB}$} & \multicolumn{2}{|c|}{$\Delta$ BDNF } & \multicolumn{2}{|c|}{$\Delta$ Klotho } & \multirow{2}{*}{$\frac{\text { UPA }}{\text { Fold of }}$} & \multirow{2}{*}{$\begin{array}{c}\text { EPA } \\
\text { change }\end{array}$} \\
\hline & & & $\mathbf{r}$ & $p$ & $\mathbf{r}$ & $p$ & $\mathbf{r}$ & $p$ & & \\
\hline \multirow[t]{9}{*}{ Amino Acid } & Tyrosine Metabolism & 3-(4-hydroxyphenyl)lactate & .137 & .533 & -.262 & .228 & .086 & .695 & 1.00 & $1.21^{*}$ \\
\hline & Methionine, Cysteine, SAM \& Taurine Metabolism & hypotaurine & -.166 & .448 & .814 & $<.001$ & .023 & .916 & 0.93 & $0.51^{*}$ \\
\hline & & taurine & -.114 & .605 & .832 & $<.001$ & .062 & .780 & 0.95 & $0.63^{*}$ \\
\hline & & methionine sulfoxide & .031 & .888 & -.332 & .121 & .120 & .586 & 0.96 & $1.14^{\star}$ \\
\hline & Tryptophan Metabolism & indolepropionate & .487 & .019 & .370 & .082 & .097 & .661 & 1.10 & $0.64 \dagger$ \\
\hline & & serotonin & -.234 & .282 & .413 & .050 & .086 & .696 & 0.21 & $0.39 \dagger$ \\
\hline & Urea Cycle; Arginine \& Proline Metabolism & n-methylproline & -.018 & .935 & .018 & .936 & .116 & .599 & 1.41 & $1.93^{*}$ \\
\hline & Phenylalanine Metabolism & phenylpyruvate & .227 & .297 & .094 & .670 & .071 & .746 & 0.87 & $0.82^{\star}$ \\
\hline & Polyamine Metabolism & spermidine & -.136 & .537 & .710 & $<.001$ & -.028 & .901 & 0.90 & $0.37^{\star}$ \\
\hline Peptide & Acetylated Peptides & phenylacetylglutamine & -.012 & .957 & -.196 & .371 & .222 & .309 & 1.01 & $1.31^{*}$ \\
\hline \multirow[t]{2}{*}{ Carbohydrate } & Aminosugar Metabolism & glucuronate & .292 & .176 & .214 & .327 & .059 & .790 & 1.08 & $1.91^{\star}$ \\
\hline & & n-acetylneuraminate & .036 & .871 & .726 & $<.001$ & .026 & .908 & 0.91 & $0.76^{\star}$ \\
\hline \multirow[t]{3}{*}{ Xenobiotics } & Benzoate Metabolism & 3-hydroxyhippurate sulfate & .329 & .125 & -.007 & .976 & .120 & .584 & 0.85 & $1.80^{*}$ \\
\hline & & 3-methoxycatechol sulfate (2) & .055 & .803 & .257 & .237 & .323 & .133 & 0.77 & $0.38^{\star}$ \\
\hline & & o-cresol sulfate & .255 & .240 & -.231 & .288 & -.022 & .922 & 1.15 & $2.43^{\star}$ \\
\hline
\end{tabular}

Analysis of associations between plasma biomarkers and non-lipid metabolites. Several metabolites display a significant correlation with BDNF (bolded $p$ values). Fold changes of the metabolites in the UPA and EPA groups is listed. Metabolites are classified by Super Pathway and Sub Pathway. ${ }^{*} p<.05 .{ }^{\dagger} .05<p<.10$. cathepsin $B$ (CTSB); brain-derived neurotrophic factor (BDNF); Usual Physical Activity (UPA); Enhanced Physical Activity (EPA).

did not demonstrate an association between CTSB and executive function (61). That study was observational and included participants with decades of exercise training experience who may have long-term adaptation and differences in CTSB signaling not captured by exercise training for 26 weeks in previously sedentary adults. In addition, these subjects participated in a variety of athletic activities. Indeed, older marathon runners have been shown to harbor elevated CTSB levels (55). It is also noteworthy our study included both males and females, and that females may drive the myokine CTSB increase and correlations with cognitive function. A potential underlying mechanism may be muscle physiology differences between females and males (62), but a larger sample would be needed to determine whether sex differences indeed play a role. Our findings help answer a call (63) for data on the effect of exercise-induced CTSB upregulation and cognition, but further studies in males and females are needed to draw stronger conclusions.

While acute exercise generally increases $\operatorname{BDNF}(15,57,64)$, effects of chronic exercise on blood BDNF levels are less clear (5, $7,12,13,15,65)$. In the present study BDNF levels decreased after exercise training and were closely linked to metabolomic alterations. Indeed, in the brain, lipid metabolites (ceramides, sphingolipids) modulate neurotrophin signaling (66-69). However, the regulation of BDNF by metabolites in the blood stream has not been examined under exercise conditions. In our study there was no association between BDNF and verbal learning and memory or executive function. Results of BDNF and cognition following exercise intervention in older adults are mixed. Some reports demonstrate no significant association between BDNF and global cognition (70) or executive function (71), and others indicate increases in both BDNF and cognition among individuals with mild cognitive impairment but do not report associations between the two outcomes (72). In contrast, a recent study demonstrated a significant relationship between plasma BDNF and improved visuospatial ability and verbal fluency, despite no absolute change in resting BDNF levels after three months of aerobic exercise training (73). However, visuospatial ability and verbal fluency are distinct from the cognitive outcomes we measured and may respond differently to BDNF. Moreover, there is some evidence to suggest that BDNF possibly only mediates exercise effects on executive function in adults over the age of 70 (74); our sample had a mean age of approximately 65 years old and thus may not have reached the age threshold needed to realize an effect of BDNF.

There was no change in serum klotho levels after 26 weeks of aerobic exercise training; however, klotho was correlated with increased cardiorespiratory fitness assessed by $\mathrm{VO}_{2}$ peak, the gold standard. Our finding is consistent with a report that klotho levels showed a weak but non-significant correlation with improvement in a proxy for cardiorespiratory fitness after three months of aerobic exercise in post-menopausal women (75). Other researchers showed that acute bouts of aerobic exercise increase klotho levels in young adults (76) and middle-aged adults of approximately 48 years (31). While older adults respond to acute aerobic exercise with increased klotho levels, the effect is attenuated in adults 65 years and older compared to those aged 25-45 years (77). It is possible that the klotho response to exercise training is similarly blunted among adults 65 years and older. This could explain the lack of change in our sample, as approximately half of our participants were older than 65 years. However, our findings contrast with reports that resting klotho levels increase after exercise training in adults with mean ages in the early 50 's and 60 's $(75,78)$. It is thus surprising that we did not observe even a modest response. The lack of correlations among klotho and cognitive outcomes in the present study was also surprising given mounting evidence that klotho is associated with multiple cognitive domains including verbal fluency (79), attention, working memory, verbal memory, executive function (80), and global cognition (81) across patient and healthy aging cohorts. However, those associations are primarily based on cross-sectional or longitudinal studies, and our results are interventional. Evidence that augmenting klotho 
A

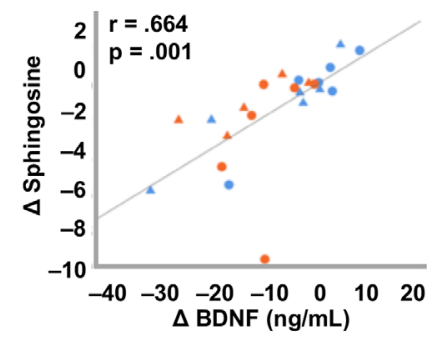

C

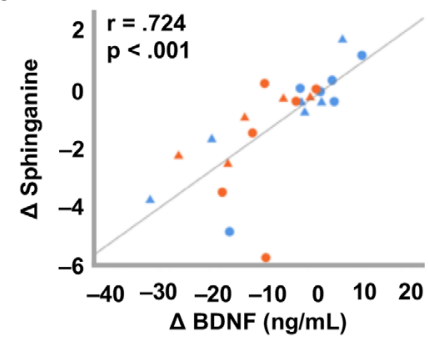

E

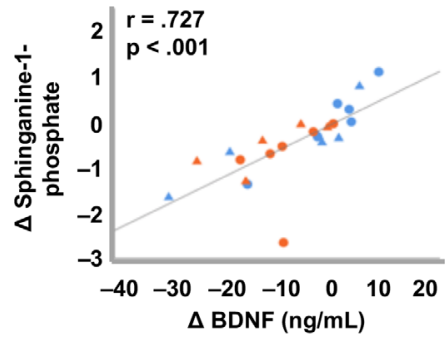

BDNF (ng/mL)
B

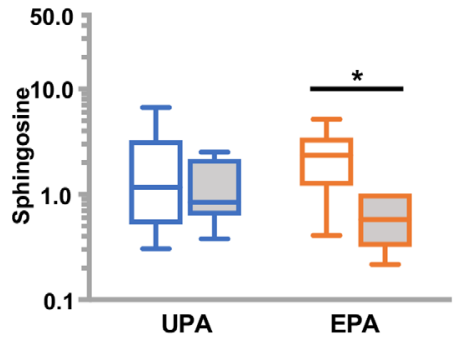

D

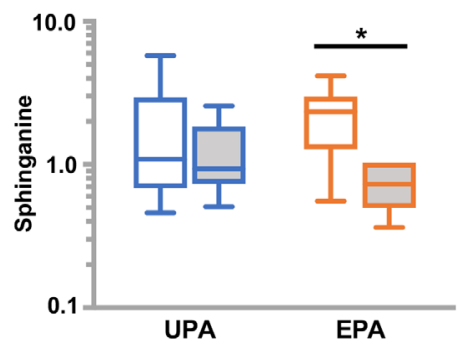

$\triangle$ UPA Male

UPA Female

EPA Male

EPA Female

$\square$ Baseline

$\square$ Post-intervention

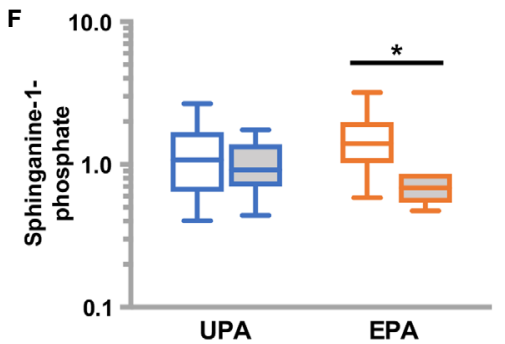

G

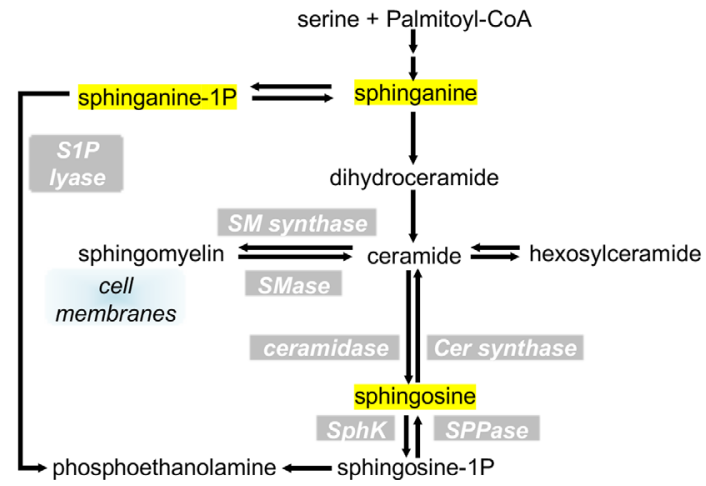

FIGURE 3 | Changes in sphingolipid metabolism and BDNF. (A, C, E) Changes in (A) sphingosine (C) spinganine and (E) sphinganine-1-phosphate correlated closely with change in BDNF. (B, D, F) Box plots showing levels of these three metabolites were reduced post-intervention in the EPA group. (G) Schematic pathway of sphingolipid metabolism. Raw metabolite values were normalized in terms of raw area counts by log transformation and rescaled to set the median equal to 1. ${ }^{*} p<.05$. brain-derived neurotrophic factor (BDNF); Usual Physical Activity (UPA); Enhanced Physical Activity (EPA).

levels can impact cognition in animal models is promising but scarce $(82,83)$, so further research is needed on whether and how exercise-induced klotho changes in older humans (78) may support cognition.

Metabolomics have become increasingly utilized to understand biochemical pathways that may be affected by AD (32-34). In particular, ceramides, sphingolipids and glycerophospholipids have been proposed as $\mathrm{AD}$ biomarkers, and have been linked to cognitive function and mood regulation $(33,84,85)$. Ceramides play a role in signaling during apoptosis, inflammation and insulin resistance, processes associated with the pathogenesis of $\mathrm{AD}(86)$. Higher ceramide $(86,87)$ and sphingomyelin $(84,85)$ levels have been detected in plasma from patients with $\mathrm{AD}$, and depression (88) and have been proposed as diagnostic indicators of these 
A
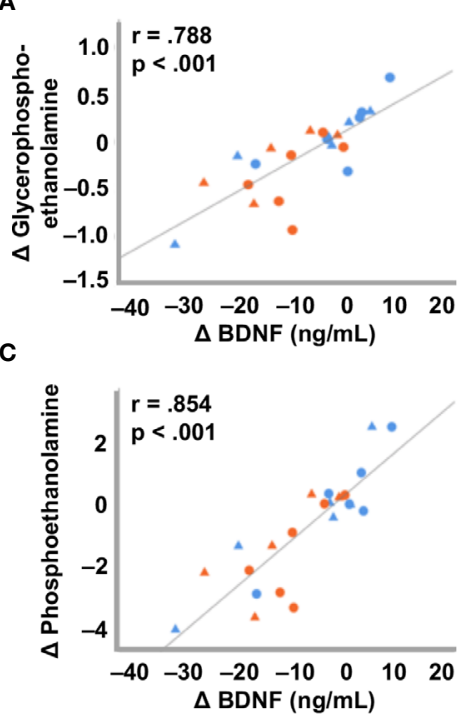

E

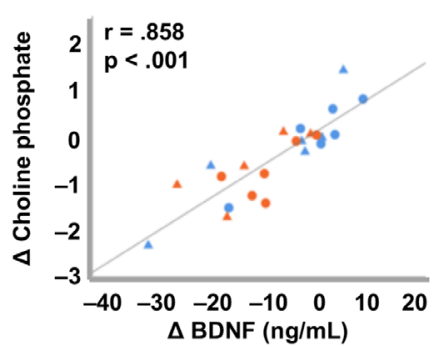

G

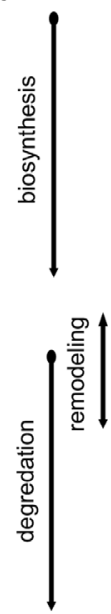

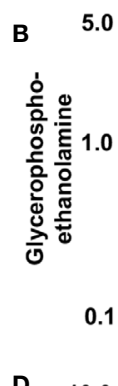

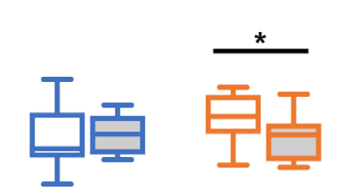

$\triangle$ UPA Male

UPA Female

$\triangle$ EPA Male

EPA Female

$\square$ Baseline

$\square$ Post-intervention

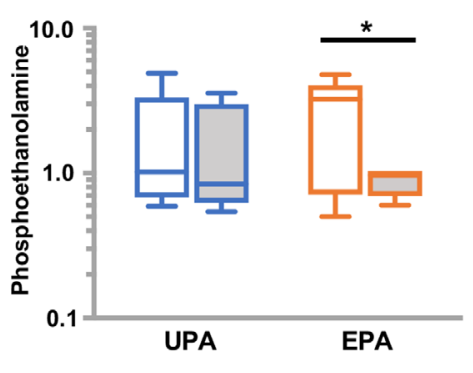

F

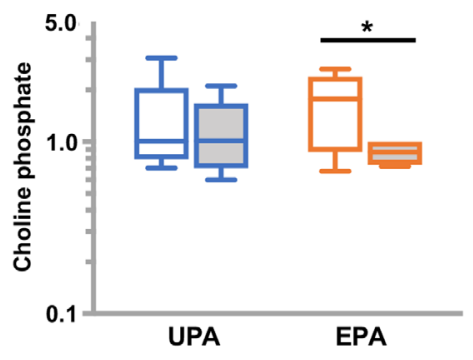

FIGURE 4 | Alterations in phospholipid metabolism and BDNF. (A, C, E) Changes in (A) glycero-phosphoethanolamine (C) phosphoethanolamine and (E) choline phosphate correlated closely with change in BDNF. (B, D, F) Box plots showing levels of these three metabolites were reduced post-intervention in the EPA group. (G) Schematic of the phospholipid metabolic pathway. Raw metabolite values were normalized in terms of raw area counts by log transformation and rescaled to set the median equal to 1. ${ }^{*} p<.05$. brain-derived neurotrophic factor (BDNF); Usual Physical Activity (UPA); Enhanced Physical Activity (EPA).

diseases. In the present study, exercise training reduced several ceramide and sphingomyelin species, and dihydrosphingomyelins. This finding is consistent with previous research showing that exercise reduced plasma ceramide (c14:0) in obese and diabetic subjects (89) and in mice (90). Exercise training also decreased sphingosines and sphingolipid synthesis metabolites. The exerciseinduced decrease in sphinganine-1-phosphate and precursor sphinganine is of interest, as elevated serum sphinganine-1- 


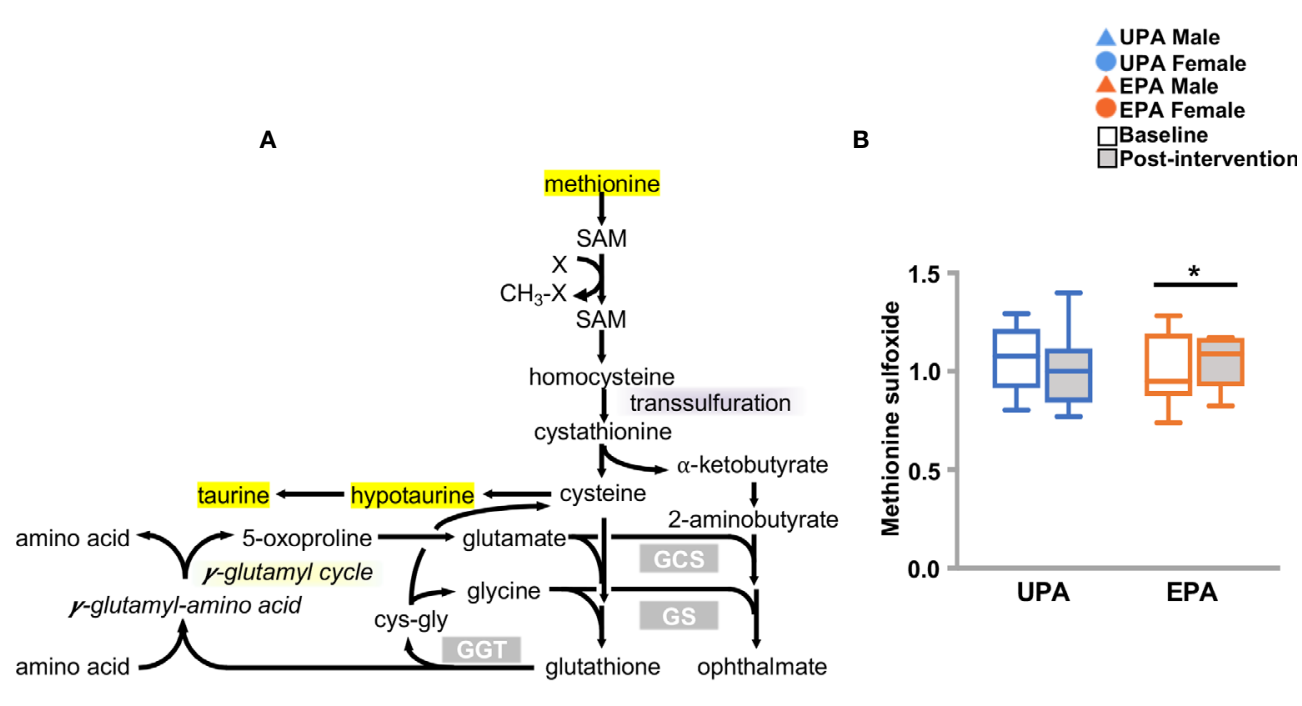

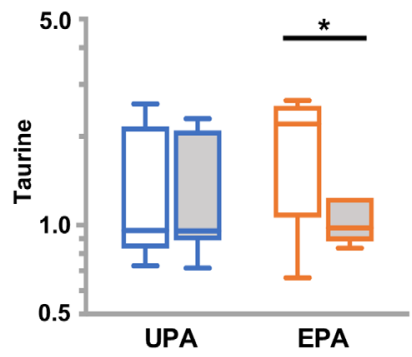

$\mathbf{E}$

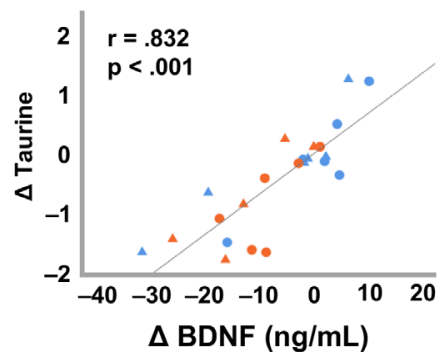

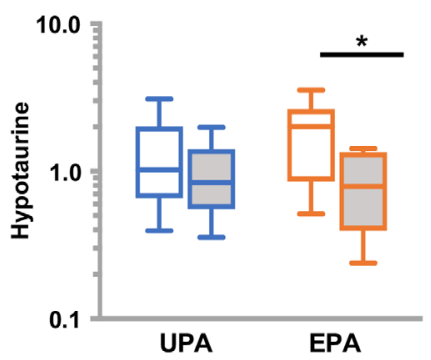

$\mathbf{F}$

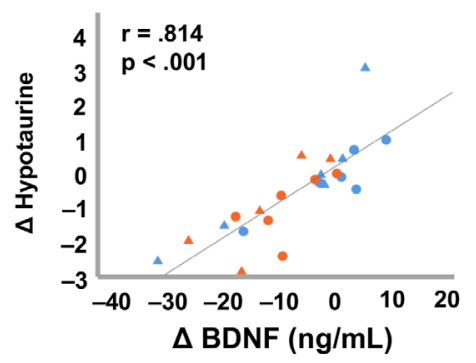

FIGURE 5 | Changes in markers of oxidative stress and BDNF. (A) Schematic of the redox pathway. (B-D) Exercise training resulted in changes in redox homeostasis with an increase in (B) methionine sulfoxide, and decreases in (C) taurine and (D) hypotaurine. Changes in (E) taurine and (F) hypotaurine were closely associated with changes in BDNF. Raw metabolite values were normalized in terms of raw area counts by log transformation and rescaled to set the median equal to 1. ${ }^{*} p<.05$. brain-derived neurotrophic factor (BDNF); Usual Physical Activity (UPA); Enhanced Physical Activity (EPA).

phosphate is associated with the conversion from MCI to AD (91). Sphingosine 1-phosphate mediates multiple physiological processes, including regulation of vascular and immune systems and is considered neuroprotective (92-94), albeit that an elevated ratio of d18:1 to d16:1 sphingosine 1-phosphate species may be pro-inflammatory (95). Exercise increased sphingosine 1phosphate in plasma in young adults (96), but is reduced during recovery (97) and after ultra-endurance races (98). The observed decrease in the EPA group is consistent with other exercise studies (99), however, the functional significance of this reduction remains unclear.
Phospholipids are key constituents of plasma membranes and can contribute to synaptic dysfunction in AD (100). Several of these were changed post-intervention in the EPA group. For instance, choline phosphate is elevated in $\mathrm{AD}$ patient cerebrospinal fluid (101) and was lowered in serum after exercise training. In addition, multiple phosphatidylethanolamines, which play a role in inflammation and apoptosis, were reduced by exercise training, consistent with research in mouse models (102). Several lysophospholipids were elevated by exercise training. This finding is of interest since lipids in this pathway are decreased in mild cognitive impairment (MCI) and $\mathrm{AD}$ 


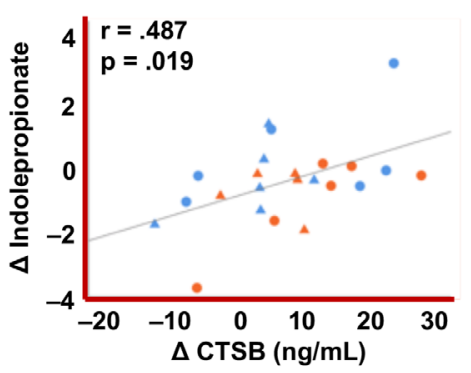

C
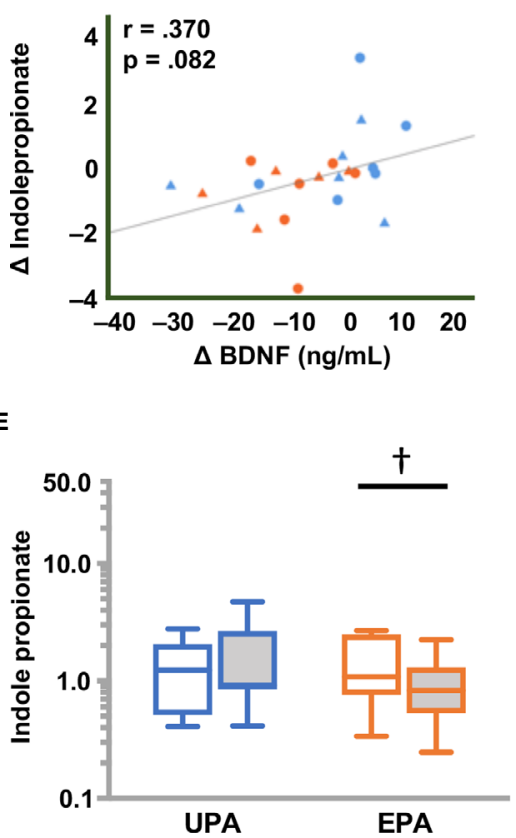

B

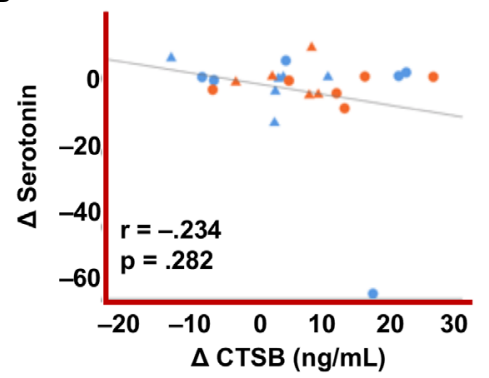

D

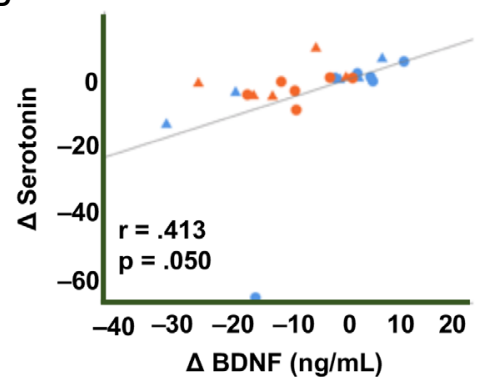

$\mathbf{F}$

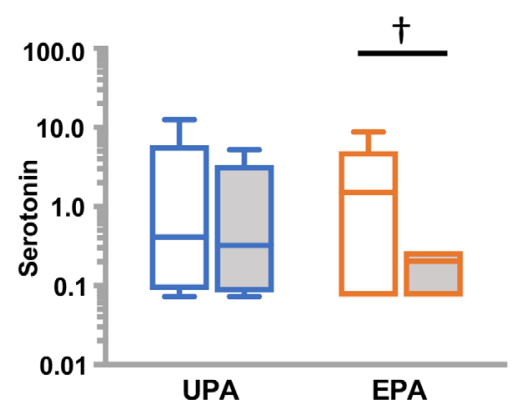

FIGURE 6 | Differences in molecules originating from the gut microbiome, and BDNF and CTSB (A) Change in CTSB was significantly associated with a change indoleproprionate (IPA), but not with (B) serotonin. (C) Change in BDNF showed a weak but non-significant correlation with IPA and (D) a significant correlation with serotonin. (E) IPA and (F) serotonin levels were lower post-intervention in the EPA group. Raw metabolite values were normalized in terms of raw area counts by log transformation and rescaled to set the median equal to $1{ }^{\dagger} .05<p<.10$. cathepsin B (CTSB); brain-derived neurotrophic factor (BDNF); Usual Physical Activity (UPA); Enhanced Physical Activity (EPA).

compared to normal controls (103). We also observed that levels of PUFAs were elevated in the EPA group. Exercise training increased omega-6 arachidonate, as well as docosapentaenoate (DPA), an omega- 6 considered to be neuroprotective $(104,105)$, and omega-3 PUFAs docosahexanoate (DHA) and dihomolinolenate (106). In the context of $\mathrm{AD}$, DHA has been shown to reduce both cognitive decline and neuroinflammation (107). It is noteworthy that there were no changes in plasma lipoproteins, such as LDL and HDL cholesterol, which transport phospholipids (46), indicating that exercise specifically induced alterations in lipid metabolite signaling. Furthermore, in the present study no correlations between cognition and changes in lipid metabolites were observed. Both lipid metabolism (108) and BDNF (109) play a role in mood disorders. Multiple phospho-and sphingolipids were tightly associated with change in BDNF levels, suggesting regulation of BDNF levels (110). As depression is linked to $\mathrm{AD}$ (111) it would be of interest in further studies to examine the association between mood, exercise, and lipid metabolomics in older adults with $\mathrm{AD}$ risk factors.

While the overall effects of exercise in this study appear to be beneficial for brain function, some aspects remain unclear. Aerobic exercise is associated with increased generation of 
reactive oxygen species, which leads to higher rates of mitochondrial oxidation (112). Increased oxidative stress induced by exercise activates an antioxidant response, which results in elevated expression of antioxidant defense enzymes (113). A redox imbalance (i.e., enhanced oxidant generation and reduced antioxidant defense) has also been associated with neurodegenerative diseases including AD. Accordingly, the dynamic interplay between reactive oxygen species formation and detoxification is involved in maintenance of redox homeostasis. In the present study, we detected higher levels of methionine sulfoxide and lower levels of (hypo) taurine in the EPA group, compared to pre-exercise measurements. Elevated methionine sulfoxide has been reported in people with AD (114), whereas taurine levels are reportedly reduced (115). Taurine exerts neuroprotective functions and acts as an antioxidant. Collectively, differences in redox active compounds that we observed after the 26-week intervention could arise from agerelated reductions in antioxidant defense or from the increased risk for $\mathrm{AD}$ in these subjects, which can result in oxidative damage of macromolecules. Whether exercise-induced oxidative stress might exert protective effects through the activation of antioxidant response mechanisms in this population remains to be determined. Spermidine is a polyamine metabolite (50) that has been implicated in cognitive function and $\operatorname{AD}(116,117)$ and may alleviate oxidative stress. Increased dietary intake of spermidine is considered beneficial for brain function in people with dementia (116). Consistent with our findings of other antioxidant metabolites, spermidine was diminished in the EPA group.

Physical exercise has been reported to positively affect microbiota diversity and activity, resulting in beneficial health effects (118). Commensal bacteria present in the gut also contribute to the host metabolism by catabolizing and producing a large variety of compounds (119). Changes in the composition of commensal bacteria have been associated with several neurological diseases, including AD (120). We found that exercise increased catabolites of phenylalanine and tyrosine, whereas it reduced levels of serotonin. Although peripheral serotonin is produced in the gut by enterochromaffin cells, gut microbiota can actively modulate the biosynthesis of this neurotransmitter by host cells (121). The reduction in plasma serotonin levels that we detected in the present study is consistent with reported changes in serotonin levels due to exercise (122), and was correlated with the change in plasma BDNF levels (123). Another tryptophan metabolite is indolepropionate (IPA). This was the only metabolite that correlated with change in CTSB. Interestingly, reduced levels, as observed in the present study after exercise, are associated with improved muscle function (124). Elevated levels are associated with inflammation in older adults (125). Collectively, these results support that the gut microbiome is dynamically involved in the anti-depressant effects of physical activity (126) and muscle function.

Several limitations should be considered when interpreting this study. The extent to which these exercise-induced changes in systemic biomarkers reflect alterations in brain function remains unknown, as in-vivo brain measurements post-exercise training are not currently possible. Nonetheless, there is evidence that peripheral BDNF and CTSB influence central levels $(25,127,128)$ and that peripheral metabolites such as sphingolipids are associated with $\mathrm{AD}$ pathology (33). It is possible that some of our findings are specific to the present study population; comparison to a population without parental history and APOE-related risk for $\mathrm{AD}$ would reinforce our conclusions by evaluating whether our findings are specific to those with these particular risk factors. Further research should determine whether the observed systemic biomarker changes also occur in the general population, and larger sample sizes are warranted based on the present findings. This study was designed to measure blood at baseline and post-intervention, so it is possible that acute changes in systemic biomarkers and metabolites (129) occurred with each exercise training session but escaped measurement. Furthermore, the mechanisms underlying metabolite-related changes in plasma BDNF $(66-68,130)$ with exercise, and whether and how this may result in increased brain levels of this neurotrophin, remains to be elucidated. Moreover, while this study extends and confirms a link between CTSB and cognition, additional older and younger study populations, and memory tests will be needed to further explore this finding. Finally, resistance exercise training may also support brain health (131); however, aerobic exercise was employed in this study due to its upregulation of CTSB and klotho $(25,31,55)$, favorable effect on cardiorespiratory fitness (36), and wellestablished link to cognitive function $(5,6,132)$.

In conclusion, plasma CTSB levels were increased following this 26-week structured aerobic exercise training in adults at risk for $\mathrm{AD}$, and change in CTSB was positively associated with cognitive function. Plasma BDNF levels decreased in conjunction with metabolomic changes. Serum klotho was unchanged but was associated with cardiorespiratory fitness. Multiple lipid metabolites relevant to $\mathrm{AD}$ were modified by exercise in a manner that may be neuroprotective. Our findings position CTSB, BDNF, and klotho as exercise biomarkers for evaluating the effect of lifestyle interventions on brain function.

\section{DATA AVAILABILITY STATEMENT}

The datasets presented in this study can be found in online repositories. The names of the repository/repositories and accession number(s) can be found below: Metabolomics Workbench, Project ID PR001121 and doi: http://doi.org/10. 21228/M8SX23.

\section{ETHICS STATEMENT}

The studies involving human participants were reviewed and approved by The University of Wisconsin Institutional Review Board. The patients/participants provided their written informed consent to participate in this study. 


\section{AUTHOR CONTRIBUTIONS}

DC, OO, and HvP designed experiments. JG, HYM, DD, and MS performed experiments and/or analyzed the data. JG, HYM, OO, and HvP drafted the manuscript. All authors revised the manuscript and approved the final version. All authors contributed to the article and approved the submitted version.

\section{FUNDING}

This work was supported by grants from the Alzheimer's Association (NIRGD-305257), the Extendicare Foundation, and the National Institutes of Health (K23 AG045957, R01 AG027161, P50 AG033514, and UL1RR025011). This work was also supported in part by the NIA Intramural Research Program, the Florida Atlantic University Brain Institute, Jupiter

\section{REFERENCES}

1. Spires-Jones TL, Hyman BT. The Intersection of Amyloid Beta and Tau at Synapses in Alzheimer's Disease. Neuron (2014) 82(4):756-71. doi: 10.1016/ j.neuron.2014.05.004

2. Okonkwo OC, Xu G, Dowling NM, Bendlin BB, Larue A, Hermann BP, et al. Family History of Alzheimer Disease Predicts Hippocampal Atrophy in Healthy Middle-Aged Adults. Neurology (2012) 78(22):1769-76. doi: 10.1212/WNL.0b013e3182583047

3. Honig LS, Vellas B, Woodward M, Boada M, Bullock R, Borrie M, et al. Trial of Solanezumab for Mild Dementia Due to Alzheimer's Disease. N Engl J Med (2018) 378(4):321-30. doi: 10.1056/NEJMoa1705971

4. Vivar C, Potter MC, van Praag H. All About Running: Synaptic Plasticity, Growth Factors and Adult Hippocampal Neurogenesis. Curr Top Behav Neurosci (2013) 15:189-210. doi: 10.1007/7854_2012_220

5. Voss MW, Soto C, Yoo S, Sodoma M, Vivar C, van Praag H. Exercise and Hippocampal Memory Systems. Trends Cognit Sci (2019) 23(4):318-33. doi: 10.1016/j.tics.2019.01.006

6. Voss MW, Erickson KI, Prakash RS, Chaddock L, Kim JS, Alves H, et al. Neurobiological Markers of Exercise-Related Brain Plasticity in Older Adults. Brain Behav Immun (2013) 28:90-9. doi: 10.1016/j.bbi.2012.10.021

7. Erickson KI, Voss MW, Prakash RS, Basak C, Szabo A, Chaddock L, et al. Exercise Training Increases Size of Hippocampus and Improves Memory. Proc Natl Acad Sci USA (2011) 108(7):3017-22. doi: 10.1073/ pnas. 1015950108

8. Maass A, Duzel S, Goerke M, Becke A, Sobieray U, Neumann K, et al. Vascular Hippocampal Plasticity After Aerobic Exercise in Older Adults. Mol Psychiatry (2015) 20(5):585-93. doi: 10.1038/mp.2014.114

9. Cotman CW, Berchtold NC, Christie LA. Exercise Builds Brain Health: Key Roles of Growth Factor Cascades and Inflammation. Trends Neurosci (2007) 30(9):464-72. doi: 10.1016/j.tins.2007.06.011

10. Voss MW, Vivar C, Kramer AF, van Praag H. Bridging Animal and Human Models of Exercise-Induced Brain Plasticity. Trends Cognit Sci (2013) 17 (10):525-44. doi: 10.1016/j.tics.2013.08.001

11. Duzel E, van Praag H, Sendtner M. Can Physical Exercise in Old Age Improve Memory and Hippocampal Function? Brain (2016) 139(Pt 3):66273. doi: 10.1093/brain/awv407

12. Babaei P, Damirchi A, Mehdipoor M, Tehrani BS. Long Term Habitual Exercise is Associated With Lower Resting Level of Serum BDNF. Neurosci Lett (2014) 566:304-8. doi: 10.1016/j.neulet.2014.02.011

13. Currie J, Ramsbottom R, Ludlow H, Nevill A, Gilder M. Cardio-Respiratory Fitness, Habitual Physical Activity and Serum Brain Derived Neurotrophic Factor (BDNF) in Men and Women. Neurosci Lett (2009) 451(2):152-5. doi: 10.1016/j.neulet.2008.12.043
Life Sciences Initiative, Florida Department of Health, Ed and Ethel Moore Alzheimer's Disease Research program.

\section{ACKNOWLEDGMENTS}

We thank Andrew Wiese for helpful comments on the manuscript. We thank the staff and study participants of the Wisconsin Registry for Alzheimer's Prevention and the Wisconsin Alzheimer's Disease Research Center.

\section{SUPPLEMENTARY MATERIAL}

The Supplementary Material for this article can be found online at: https://www.frontiersin.org/articles/10.3389/fendo.2021. 660181/full\#supplementary-material

14. da Silva Germanos S, Vieira B, Reichert Vital da Silva I, da Cunha JJ, Nique S Striebel V, et al. The Impact of an Aquatic Exercise Program on BDNF Levels in Parkinson's Disease Patients: Short-and Long-Term Outcomes. Funct Neurol (2019) 34(2):65-70.

15. Figueiredo C, Antunes BM, Giacon TR, Vanderlei LCM, Campos EZ, Peres $\mathrm{FP}$, et al. Influence of Acute and Chronic High-Intensity Intermittent Aerobic Plus Strength Exercise on BDNF, Lipid and Autonomic Parameters. J Sports Sci Med (2019) 18(2):359-68.

16. Huang T, Larsen KT, Ried-Larsen M, Moller NC, Andersen LB. The Effects of Physical Activity and Exercise on Brain-Derived Neurotrophic Factor in Healthy Humans: A Review. Scand J Med Sci Sports (2014) 24(1):1-10. doi: $10.1111 /$ sms.12069

17. Jung SH, Kim J, Davis JM, Blair SN, Cho HC. Association Among Basal Serum BDNF, Cardiorespiratory Fitness and Cardiovascular Disease Risk Factors in Untrained Healthy Korean Men. Eur J Appl Physiol (2011) 111 (2):303-11. doi: 10.1007/s00421-010-1658-5

18. Pedersen BK, Febbraio MA. Muscle as an Endocrine Organ: Focus on Muscle-Derived Interleukin-6. Physiol Rev (2008) 88(4):1379-406. doi: 10.1152/physrev.90100.2007

19. Lal C, Hardiman G, Kumbhare S, Strange C. Proteomic Biomarkers of Cognitive Impairment in Obstructive Sleep Apnea Syndrome. Sleep Breath (2019) 23(1):251-7. doi: 10.1007/s11325-018-1693-8

20. Long Z, Chen J, Zhao Y, Zhou W, Yao Q, Wang Y, et al. Dynamic Changes of Autophagic Flux Induced by Abeta in the Brain of Postmortem Alzheimer's Disease Patients, Animal Models and Cell Models. Aging (Albany NY) (2020) 12(11):10912-30. doi: 10.18632/aging.103305

21. Embury CM, Dyavarshetty B, Lu Y, Wiederin JL, Ciborowski P, Gendelman $\mathrm{HE}$, et al. Cathepsin B Improves Ss-Amyloidosis and Learning and Memory in Models of Alzheimer's Disease. J Neuroimmune Pharmacol (2017) 12 (2):340-52. doi: 10.1007/s11481-016-9721-6

22. Mueller-Steiner S, Zhou Y, Arai H, Roberson ED, Sun B, Chen J, et al Antiamyloidogenic and Neuroprotective Functions of Cathepsin B: Implications for Alzheimer's Disease. Neuron (2006) 51(6):703-14. doi: 10.1016/j.neuron.2006.07.027

23. Hook G, Hook V, Kindy M. The Cysteine Protease Inhibitor, E64d, Reduces Brain Amyloid-Beta and Improves Memory Deficits in Alzheimer's Disease Animal Models by Inhibiting Cathepsin B, But Not BACE1, Beta-Secretase Activity. J Alzheimers Dis (2011) 26(2):387-408. doi: 10.3233/JAD-2011110101

24. Hook VY, Kindy M, Hook G. Inhibitors of Cathepsin B Improve Memory and Reduce Beta-Amyloid in Transgenic Alzheimer Disease Mice Expressing the Wild-Type, But Not the Swedish Mutant, Beta-Secretase Site of the Amyloid Precursor Protein. J Biol Chem (2008) 283(12):7745-53 doi: 10.1074/jbc.M708362200 
25. Moon HY, Becke A, Berron D, Becker B, Sah N, Benoni G, et al. RunningInduced Systemic Cathepsin B Secretion is Associated With Memory Function. Cell Metab (2016) 24(2):332-40. doi: 10.1016/j.cmet.2016.05.025

26. Dubal DB, Yokoyama JS, Zhu L, Broestl L, Worden K, Wang D, et al. Life Extension Factor Klotho Enhances Cognition. Cell Rep (2014) 7(4):1065-76. doi: 10.1016/j.celrep.2014.03.076

27. Dubal DB, Zhu L, Sanchez PE, Worden K, Broestl L, Johnson E, et al. Life Extension Factor Klotho Prevents Mortality and Enhances Cognition in Happ Transgenic Mice. J Neurosci (2015) 35(6):2358-71. doi: 10.1523/ JNEUROSCI.5791-12.2015

28. Leon J, Moreno AJ, Garay BI, Chalkley RJ, Burlingame AL, Wang D, et al. Peripheral Elevation of a Klotho Fragment Enhances Brain Function and Resilience in Young, Aging, and Alpha-Synuclein Transgenic Mice. Cell Rep (2017) 20(6):1360-71. doi: 10.1016/j.celrep.2017.07.024

29. Erickson CM, Schultz SA, Oh JM, Darst BF, Ma Y, Norton D, et al. KLOTHO Heterozygosity Attenuates APOE4-Related Amyloid Burden in Preclinical AD. Neurology (2019) 92(16):e1878-89. doi: 10.1212/ wnl.0000000000007323

30. Ji N, Luan J, Hu F, Zhao Y, Lv B, Wang W, et al. Aerobic Exercise-Stimulated Klotho Upregulation Extends Life Span by Attenuating the Excess Production of Reactive Oxygen Species in the Brain and Kidney. Exp Ther Med (2018) 16(4):3511-7. doi: 10.3892/etm.2018.6597

31. Tan SJ, Chu MM, Toussaint ND, Cai MM, Hewitson TD, Holt SG. HighIntensity Physical Exercise Increases Serum Alpha-Klotho Levels in Healthy Volunteers. J Circ Biomark (2018) 7:1849454418794582. doi: 10.1177/ 1849454418794582

32. Pena-Bautista C, Roca M, Hervas D, Cuevas A, Lopez-Cuevas R, Vento M, et al. Plasma Metabolomics in Early Alzheimer's Disease Patients Diagnosed With Amyloid Biomarker. J Proteomics (2019) 200:144-52. doi: 10.1016/ j.jprot.2019.04.008

33. Varma VR, Oommen AM, Varma S, Casanova R, An Y, Andrews RM, et al. Brain and Blood Metabolite Signatures of Pathology and Progression in Alzheimer Disease: A Targeted Metabolomics Study. PloS Med (2018) 15(1): e1002482. doi: 10.1371/journal.pmed.1002482

34. Hurtado MO, Kohler I, de Lange EC. Next-Generation Biomarker Discovery in Alzheimer's Disease Using Metabolomics - From Animal to Human Studies. Bioanalysis (2018) 10(18):1525-46. doi: 10.4155/bio-2018-0135

35. Gaitán JM, Boots EA, Dougherty RJ, Ma Y, Edwards DF, Mitchell CC, et al. Protocol of Aerobic Exercise and Cognitive Health (REACH): A Pilot Study. J Alzheimers Dis Rep (2020) 4(1):107-21. doi: 10.3233/ADR-200180

36. Gaitán JM, Boots EA, Dougherty RJ, Oh JM, Ma Y, Edwards DF, et al. Brain Glucose Metabolism, Cognition, and Cardiorespiratory Fitness Following Exercise Training in Adults at Risk for Alzheimer's Disease. Brain Plast (2019) 5(1):83-95. doi: 10.3233/BPL-190093

37. American College of Sports Medicine. Acsm's Guidelines for Exercise Testing and Prescription. Philadelphia: Wolters Kluwer (2018).

38. Folstein MF, Folstein SE, McHugh PR. "Mini-Mental State". A Practical Method for Grading the Cognitive State of Patients for the Clinician. J Psychiatr Res (1975) 12(3):189-98. doi: 10.1016/0022-3956(75)90026-6

39. Delis D, Kramer J, Kaplan E, Ober B. California Verbal Learning Test Second Edition. San Antonio, TX: Psychological Corporation (2000).

40. Delis DC, Kaplan E, Kramer JH. Delis-Kaplan Executive Function System. San Antonio, TX: Psychological Corporation (2001).

41. Harrington MG, Chiang J, Pogoda JM, Gomez M, Thomas K, Marion SD, et al. Executive Function Changes Before Memory in Preclinical Alzheimer's Pathology: A Prospective, Cross-Sectional, Case Control Study. PloS One (2013) 8(11):e79378. doi: 10.1371/journal.pone.0079378

42. Yamazaki Y, Imura A, Urakawa I, Shimada T, Murakami J, Aono Y, et al. Establishment of Sandwich ELISA for Soluble Alpha-Klotho Measurement: Age-Dependent Change of Soluble Alpha-Klotho Levels in Healthy Subjects. Biochem Biophys Res Commun (2010) 398(3):513-8. doi: 10.1016/ j.bbrc.2010.06.110

43. Prather AA, Epel ES, Arenander J, Broestl L, Garay BI, Wang D, et al. Longevity Factor Klotho and Chronic Psychological Stress. Transl Psychiatry (2015) 5:e585. doi: 10.1038/tp.2015.81

44. Di Pardo A, Maglione V. Sphingolipid Metabolism: A New Therapeutic Opportunity for Brain Degenerative Disorders. Front Neurosci (2018) 12:249. doi: $10.3389 /$ fnins.2018.00249
45. Hannun YA, Obeid LM. Sphingolipids and Their Metabolism in Physiology and Disease. Nat Rev Mol Cell Biol (2018) 19(3):175-91. doi: 10.1038/ nrm.2017.107

46. Dashti M, Kulik W, Hoek F, Veerman EC, Peppelenbosch MP, Rezaee F. A Phospholipidomic Analysis of All Defined Human Plasma Lipoproteins. Sci Rep (2011) 1:139. doi: 10.1038/srep00139

47. Arifin SA, Falasca M. Lysophosphatidylinositol Signalling and Metabolic Diseases. Metabolites (2016) 6(1):6. doi: 10.3390/metabo6010006

48. Medina-Vera D, Rosell-Valle C, López-Gambero AJ, Navarro JA, Zambrana-Infantes EN, Rivera P, et al. Imbalance of Endocannabinoid/ Lysophosphatidylinositol Receptors Marks the Severity of Alzheimer's Disease in a Preclinical Model: A Therapeutic Opportunity. Biol (Basel) (2020) 9(11):377. doi: 10.3390/biology9110377

49. Sun GY, Simonyi A, Fritsche KL, Chuang DY, Hannink M, Gu Z, et al. Docosahexaenoic Acid (DHA): An Essential Nutrient and a Nutraceutical for Brain Health and Diseases. Prostaglandins Leukot Essent Fatty Acids (2018) 136:3-13. doi: 10.1016/j.plefa.2017.03.006

50. Madeo F, Eisenberg T, Pietrocola F, Kroemer G. Spermidine in Health and Disease. Science (2018) 359(6374):3674. doi: 10.1126/science.aan2788

51. Lavefve L, Howard LR, Carbonero F. Berry Polyphenols Metabolism and Impact on Human Gut Microbiota and Health. Food Funct (2020) 11(1):4565. doi: $10.1039 / \mathrm{c} 9$ fo0 $01634 \mathrm{a}$

52. Wu G. Important Roles of Dietary Taurine, Creatine, Carnosine, Anserine and 4-Hydroxyproline in Human Nutrition and Health. Amino Acids (2020) 52(3):329-60. doi: 10.1007/s00726-020-02823-6

53. Nagata Y, Hirayama A, Ikeda S, Shirahata A, Shoji F, Maruyama M, et al. Comparative Analysis of Cerebrospinal Fluid Metabolites in Alzheimer's Disease and Idiopathic Normal Pressure Hydrocephalus in a Japanese Cohort. Biomark Res (2018) 6:5. doi: 10.1186/s40364-018-0119-x

54. Kim B, Kang S. Regular Leisure-Time Physical Activity is Effective in Boosting Neurotrophic Factors and Alleviating Menopause Symptoms. Int J Environ Res Public Health (2020) 17(22):8624. doi: 10.3390/ijerph17228624

55. Mucher P, Batmyagmar D, Perkmann T, Repl M, Radakovics A, PonocnySeliger E, et al. Basal Myokine Levels are Associated With Quality of Life and Depressed Mood in Older Adults. Psychophysiology (2021) 58(5):e13799. doi: $10.1111 /$ psyp. 13799

56. Micielska K, Kortas JA, Gmiat A, Jaworska J, Kozlowska M, LysakRadomska A, et al. Habitually Inactive Physically - a Proposed Procedure of Counteracting Cognitive Decline in Women With Diminished Insulin Sensitivity Through a High-Intensity Circuit Training Program. Physiol Behav (2020) 229:113235. doi: 10.1016/j.physbeh.2020.113235

57. Nicolini C, Michalski B, Toepp SL, Turco CV, D'Hoine T, Harasym D, et al. A Single Bout of High-Intensity Interval Exercise Increases Corticospinal Excitability, Brain-Derived Neurotrophic Factor, and Uncarboxylated Osteolcalcin in Sedentary, Healthy Males. Neuroscience (2020) 437:242-55. doi: 10.1016/j.neuroscience.2020.03.042

58. Johnson TK, Belcher DJ, Sousa CA, Carzoli JP, Visavadiya NP, Khamoui $\mathrm{AV}$, et al. Low-Volume Acute Multi-Joint Resistance Exercise Elicits a Circulating Brain-Derived Neurotrophic Factor Response But Not a Cathepsin B Response in Well-Trained Men. Appl Physiol Nutr Metab (2020) 45(12):1332-8. doi: 10.1139/apnm-2019-0854

59. Pena GS, Paez HG, Johnson TK, Halle JL, Carzoli JP, Visavadiya NP, et al. Hippocampal Growth Factor and Myokine Cathepsin B Expression Following Aerobic and Resistance Training in 3xtg-AD Mice. Int $J$ Chronic Dis (2020) 2020:5919501-. doi: 10.1155/2020/5919501

60. Lange KL, Bondi MW, Salmon DP, Galasko D, Delis DC, Thomas RG, et al. Decline in Verbal Memory During Preclinical Alzheimer's Disease: Examination of the Effect of APOE Genotype. J Int Neuropsychol Soc (2002) 8(7):943-55. doi: 10.1017/s1355617702870096

61. De la Rosa A, Solana E, Corpas R, Bartrés-Faz D, Pallàs M, Vina J, et al. Long-Term Exercise Training Improves Memory in Middle-Aged Men and Modulates Peripheral Levels of BDNF and Cathepsin B. Sci Rep (2019) 9 (1):3337. doi: 10.1038/s41598-019-40040-8

62. Rosa-Caldwell ME, Greene NP. Muscle Metabolism and Atrophy: Let's Talk About Sex. Biol Sex Differ (2019) 10(1):43. doi: 10.1186/s13293-019-0257-3

63. Kim S, Choi JY, Moon S, Park DH, Kwak HB, Kang JH. Roles of Myokines in Exercise-Induced Improvement of Neuropsychiatric Function. Pflugers Arch (2019) 471(3):491-505. doi: 10.1007/s00424-019-02253-8 
64. Cho HC, Kim J, Kim S, Son YH, Lee N, Jung SH. The Concentrations of Serum, Plasma and Platelet BDNF are All Increased by Treadmill VO(2)Max Performance in Healthy College Men. Neurosci Lett (2012) 519(1):78-83. doi: 10.1016/j.neulet.2012.05.025

65. Damirchi A, Tehrani BS, Alamdari KA, Babaei P. Influence of Aerobic Training and Detraining on Serum BDNF, Insulin Resistance, and Metabolic Risk Factors in Middle-Aged Men Diagnosed With Metabolic Syndrome. Clin J Sport Med (2014) 24(6):513-8. doi: 10.1097/jsm.0000000000000082

66. Crivelli SM, Giovagnoni C, Visseren L, Scheithauer AL, de Wit N, den Hoedt S, et al. Sphingolipids in Alzheimer's Disease, How can We Target Them? Adv Drug Delivery Rev (2020) 159:214-31. doi: 10.1016/j.addr.2019.12.003

67. Deogracias R, Yazdani M, Dekkers MP, Guy J, Ionescu MC, Vogt KE, et al. Fingolimod, a Sphingosine-1 Phosphate Receptor Modulator, Increases BDNF Levels and Improves Symptoms of a Mouse Model of Rett Syndrome. Proc Natl Acad Sci USA (2012) 109(35):14230-5. doi: 10.1073/pnas.1206093109

68. Dobrowsky RT, Werner MH, Castellino AM, Chao MV, Hannun YA. Activation of the Sphingomyelin Cycle Through the Low-Affinity Neurotrophin Receptor. Science (1994) 265(5178):1596-9. doi: 10.1126/ science. 8079174

69. Luther JA, Enes J, Birren SJ. Neurotrophins Regulate Cholinergic Synaptic Transmission in Cultured Rat Sympathetic Neurons Through a P75-Dependent Mechanism. J Neurophysiol (2013) 109(2):485-96. doi: 10.1152/jn.00076.2011

70. Rezola-Pardo C, Hervas G, Arrieta H, Hernandez-de Diego A, Ruiz-Litago F, Gil SM, et al. Physical Exercise Interventions Have No Effect on Serum BDNF Concentration in Older Adults Living in Long-Term Nursing Homes. Exp Gerontol (2020) 139:111024. doi: 10.1016/j.exger.2020.111024

71. Grégoire CA, Berryman N, St-Onge F, Vu TTM, Bosquet L, Arbour N, et al. Gross Motor Skills Training Leads to Increased Brain-Derived Neurotrophic Factor Levels in Healthy Older Adults: A Pilot Study. Front Physiol (2019) 10:410. doi: 10.3389/fphys.2019.00410

72. Nascimento CM, Pereira JR, Pires de Andrade L, Garuffi M, Ayan C, Kerr DS, et al. Physical Exercise Improves Peripheral BDNF Levels and Cognitive Functions in Mild Cognitive Impairment Elderly With Different Bdnf Val66Met Genotypes. J Alzheimers Dis (2015) 43(1):81-91. doi: 10.3233/ JAD-140576

73. Máderová D, Krumpolec P, Slobodova L, Schon M, Tirpakova V, Kovanicova Z, et al. Acute and Regular Exercise Distinctly Modulate Serum, Plasma and Skeletal Muscle BDNF in the Elderly. Neuropeptides (2019) 78:101961. doi: 10.1016/j.npep.2019.101961

74. Leckie RL, Oberlin LE, Voss MW, Prakash RS, Szabo-Reed A, ChaddockHeyman L, et al. BDNF Mediates Improvements in Executive Function Following a 1-Year Exercise Intervention. Front Hum Neurosci (2014) 8:985. doi: 10.3389 /fnhum.2014.00985

75. Matsubara T, Miyaki A, Akazawa N, Choi Y, Ra SG, Tanahashi K, et al. Aerobic Exercise Training Increases Plasma Klotho Levels and Reduces Arterial Stiffness in Postmenopausal Women. Am J Physiol Heart Circ Physiol (2014) 306(3):H348-55. doi: 10.1152/ajpheart.00429.2013

76. Mostafidi E, Moeen A, Nasri H, Ghorbani Hagjo A, Ardalan M. Serum Klotho Levels in Trained Athletes. Nephrourol Mon (2016) 8(1):e30245. doi: 10.5812/numonthly.30245

77. Avin KG, Coen PM, Huang W, Stolz DB, Sowa GA, Dube JJ, et al. Skeletal Muscle as a Regulator of the Longevity Protein, Klotho. Front Physiol (2014) 5:189. doi: $10.3389 /$ fphys.2014.00189

78. Amaro-Gahete FJ, De-la OA, Jurado-Fasoli L, Espuch-Oliver A, de Haro T, Gutierrez A, et al. Exercise Training Increases the s-Klotho Plasma Levels in Sedentary Middle-Aged Adults: A Randomised Controlled Trial. The FITAGEING Study. J Sports Sci (2019) 37(19):2175-83. doi: 10.1080/ 02640414.2019 .1626048

79. Wei H, Li H, Song X, Du X, Cai Y, Li C, et al. Serum Klotho: A Potential Predictor of Cerebrovascular Disease in Hemodialysis Patients. BMC Nephrol (2019) 20(1):63. doi: 10.1186/s12882-019-1232-2

80. Xiong JW, Zhan JQ, Luo T, Chen HB, Wan QG, Wang Y, et al. Increased Plasma Level of Longevity Protein Klotho as a Potential Indicator of Cognitive Function Preservation in Patients With Schizophrenia. Front Neurosci (2020) 14:610. doi: 10.3389/fnins.2020.00610

81. Shardell M, Semba RD, Rosano C, Kalyani RR, Bandinelli S, Chia CW, et al. Plasma Klotho and Cognitive Decline in Older Adults: Findings From the
Inchianti Study. J Gerontol A Biol Sci Med Sci (2016) 71(5):677-82. doi: 10.1093/gerona/glv140

82. Zeng CY, Yang TT, Zhou HJ, Zhao Y, Kuang X, Duan W, et al. Lentiviral Vector-Mediated Overexpression of Klotho in the Brain Improves Alzheimer's Disease-Like Pathology and Cognitive Deficits in Mice. Neurobiol Aging (2019) 78:18-28. doi: 10.1016/j.neurobiolaging.2019.02.003

83. Zhao Y, Zeng CY, Li XH, Yang TT, Kuang X, Du JR. Klotho Overexpression Improves Amyloid-Beta Clearance and Cognition in the APP/PS1 Mouse Model of Alzheimer's Disease. Aging Cell (2020) 19:e13239. doi: 10.1111/acel.13239

84. Mielke MM, Bandaru VV, Haughey NJ, Rabins PV, Lyketsos CG, Carlson MC. Serum Sphingomyelins and Ceramides are Early Predictors of Memory Impairment. Neurobiol Aging (2010) 31(1):17-24. doi: 10.1016/ j.neurobiolaging.2008.03.011

85. Trushina E, Mielke MM. Recent Advances in the Application of Metabolomics to Alzheimer's Disease. Biochim Biophys Acta (2014) 1842 (8):1232-9. doi: 10.1016/j.bbadis.2013.06.014

86. Olsen ASB, Faergeman NJ. Sphingolipids: Membrane Microdomains in Brain Development, Function and Neurological Diseases. Open Biol (2017) 7(5):170069. doi: 10.1098/rsob.170069

87. Han X, Rozen S, Boyle SH, Hellegers C, Cheng H, Burke JR, et al. Metabolomics in Early Alzheimer's Disease: Identification of Altered Plasma Sphingolipidome Using Shotgun Lipidomics. PloS One (2011) 6 (7):e21643. doi: 10.1371/journal.pone.0021643

88. Gracia-Garcia P, Rao V, Haughey NJ, Bandaru VV, Smith G, Rosenberg PB, et al. Elevated Plasma Ceramides in Depression. J Neuropsychiatry Clin Neurosci (2011) 23(2):215-8. doi: 10.1176/appi.neuropsych.23.2.215

89. Kasumov T, Solomon TP, Hwang C, Huang H, Haus JM, Zhang R, et al. Improved Insulin Sensitivity After Exercise Training is Linked to Reduced Plasma C14:0 Ceramide in Obesity and Type 2 Diabetes. Obes (Silver Spring) (2015) 23(7):1414-21. doi: 10.1002/oby.21117

90. Kim KU, Yoon KJ, Park S, Lee JC, Moon HY, Moon MH. Exercise-Induced Recovery of Plasma Lipids Perturbed by Ageing With Nanoflow UHPLCESI-MS/MS. Anal Bioanal Chem (2020) 412(28):8003-14. doi: 10.1007/ s00216-020-02933-w

91. Liang Q, Liu H, Zhang T, Jiang Y, Xing H, Zhang a-H. Metabolomics-Based Screening of Salivary Biomarkers for Early Diagnosis of Alzheimer's Disease. RSC Adv (2015) 5(116):96074-9. doi: 10.1039/C5RA19094K

92. Cartier A, Hla T. Sphingosine 1-Phosphate: Lipid Signaling in Pathology and Therapy. Science (2019) 366(6463):eaar5551. doi: 10.1126/science.aar5551

93. Ceccom J, Loukh N, Lauwers-Cances V, Touriol C, Nicaise Y, Gentil C, et al. Reduced Sphingosine Kinase-1 and Enhanced Sphingosine 1-Phosphate Lyase Expression Demonstrate Deregulated Sphingosine 1-Phosphate Signaling in Alzheimer's Disease. Acta Neuropathol Commun (2014) 2:12. doi: 10.1186/2051-5960-2-12

94. Fyrst H, Saba JD. An Update on Sphingosine-1-Phosphate and Other Sphingolipid Mediators. Nat Chem Biol (2010) 6(7):489-97. doi: 10.1038/ nchembio. 392

95. Chua XY, Chai YL, Chew WS, Chong JR, Ang HL, Xiang P, et al. Immunomodulatory Sphingosine-1-Phosphates as Plasma Biomarkers of Alzheimer's Disease and Vascular Cognitive Impairment. Alzheimers Res Ther (2020) 12(1):122. doi: 10.1186/s13195-020-00694-3

96. Ksiazek M, Charmas M, Klusiewicz A, Zabielski P, Dlugolecka B, Chabowski A, et al. Endurance Training Selectively Increases High-Density LipoproteinBound Sphingosine-1-Phosphate in the Plasma. Scand J Med Sci Sports (2018) 28(1):57-64. doi: 10.1111/sms.12910

97. Baranowski M, Blachnio-Zabielska AU, Charmas M, Helge JW, Dela F, Ksiazek $\mathrm{M}$, et al. Exercise Increases Sphingoid Base-1-Phosphate Levels in Human Blood and Skeletal Muscle in a Time- and Intensity-Dependent Manner. Eur J Appl Physiol (2015) 115(5):993-1003. doi: 10.1007/s00421-014-3080-x

98. Baranowski M, Gorski J, Klapcinska B, Waskiewicz Z, Sadowska-Krepa E. Ultramarathon Run Markedly Reduces Plasma Sphingosine-1-Phosphate Concentration. Int J Sport Nutr Exerc Metab (2014) 24(2):148-56. doi: 10.1123/ijsnem.2013-0093

99. Schranner D, Kastenmüller G, Schönfelder M, Römisch-Margl W, Wackerhage H. Metabolite Concentration Changes in Humans After a Bout of Exercise: A Systematic Review of Exercise Metabolomics Studies. Sports Med Open (2020) 6(1):11. doi: 10.1186/s40798-020-0238-4 
100. Bennett SA, Valenzuela N, Xu H, Franko B, Fai S, Figeys D. Using Neurolipidomics to Identify Phospholipid Mediators of Synaptic (Dys) Function in Alzheimer's Disease. Front Physiol (2013) 4:168. doi: 10.3389/ fphys.2013.00168

101. Walter A, Korth U, Hilgert M, Hartmann J, Weichel O, Hilgert M, et al. Glycerophosphocholine is Elevated in Cerebrospinal Fluid of Alzheimer Patients. Neurobiol Aging (2004) 25(10):1299-303. doi: 10.1016/j.neurobiolaging.2004.02.016

102. Zhao T, Fang F, Wang H, Lv C, Han M, Zhang Z, et al. Effect of Aerobic Exercise on Serum Metabolites in Mice With Hepatocellular Carcinoma After Surgery. Med Sci Monit (2019) 25:3181-9. doi: 10.12659/MSM.913377

103. Sato Y, Nakamura T, Aoshima K, Oda Y. Quantitative and Wide-Ranging Profiling of Phospholipids in Human Plasma by Two-Dimensional Liquid Chromatography/Mass Spectrometry. Anal Chem (2010) 82(23):9858-64. doi: $10.1021 / \mathrm{ac} 102211 \mathrm{r}$

104. Dennis EA, Norris PC. Eicosanoid Storm in Infection and Inflammation. Nat Rev Immunol (2015) 15(8):511-23. doi: 10.1038/nri3859

105. Sinclair AJ. Docosahexaenoic Acid and the Brain- What is its Role? Asia Pac J Clin Nutr (2019) 28(4):675-88. doi: 10.6133/apjcn.201912_28(4).0002

106. Shahidi F, Ambigaipalan P. Omega-3 Polyunsaturated Fatty Acids and Their Health Benefits. Annu Rev Food Sci Technol (2018) 9:345-81. doi: 10.1146/ annurev-food-111317-095850

107. Devassy JG, Leng S, Gabbs M, Monirujjaman M, Aukema HM. Omega-3 Polyunsaturated Fatty Acids and Oxylipins in Neuroinflammation and Management of Alzheimer Disease. Adv Nutr (2016) 7(5):905-16. doi: 10.3945/an.116.012187

108. Liu L, Zhou X, Zhang Y, Liu Y, Yang L, Pu J, et al. The Identification of Metabolic Disturbances in the Prefrontal Cortex of the Chronic Restraint Stress Rat Model of Depression. Behav Brain Res (2016) 305:148-56. doi: 10.1016/j.bbr.2016.03.005

109. Duman RS, Monteggia LM. A Neurotrophic Model for Stress-Related Mood Disorders. Biol Psychiatry (2006) 59(12):1116-27. doi: 10.1016/ j.biopsych.2006.02.013

110. Kotlega D, Zembron-Lacny A, Golab-Janowska M, Nowacki P, Szczuko M. The Association of Free Fatty Acids and Eicosanoids With the Severity of Depressive Symptoms in Stroke Patients. Int J Mol Sci (2020) 21(15):5220. doi: $10.3390 /$ ijms 21155220

111. Dafsari FS, Jessen F. Depression-an Underrecognized Target for Prevention of Dementia in Alzheimer's Disease. Transl Psychiatry (2020) 10(1):160. doi: 10.1038/s41398-020-0839-1

112. Powers SK, Deminice R, Ozdemir M, Yoshihara T, Bomkamp MP, Hyatt H. Exercise-Induced Oxidative Stress: Friend or Foe? J Sport Health Sci (2020) 9 (5):415-25. doi: 10.1016/j.jshs.2020.04.001

113. Radak Z, Ishihara K, Tekus E, Varga C, Posa A, Balogh L, et al. Exercise, Oxidants, and Antioxidants Change the Shape of the Bell-Shaped Hormesis Curve. Redox Biol (2017) 12:285-90. doi: 10.1016/j.redox.2017.02.015

114. Ringman JM, Fithian AT, Gylys K, Cummings JL, Coppola G, Elashoff D, et al. Plasma Methionine Sulfoxide in Persons With Familial Alzheimer's Disease Mutations. Dement Geriatr Cognit Disord (2012) 33(4):219-25. doi: $10.1159 / 000338546$

115. Jakaria M, Azam S, Haque ME, Jo SH, Uddin MS, Kim IS, et al. Taurine and its Analogs in Neurological Disorders: Focus on Therapeutic Potential and Molecular Mechanisms. Redox Biol (2019) 24:101223. doi: 10.1016/j.redox. 2019.101223

116. Pekar T, Wendzel A, Flak W, Kremer A, Pauschenwein-Frantsich S, Gschaider A, et al. Spermidine in Dementia: Relation to Age and Memory Performance. Wien Klin Wochenschr (2020) 132(1-2):42-6. doi: 10.1007/ s00508-019-01588-7

117. Wirth M, Benson G, Schwarz C, Kobe T, Grittner U, Schmitz D, et al. The Effect of Spermidine on Memory Performance in Older Adults at Risk for Dementia: A Randomized Controlled Trial. Cortex (2018) 109:181-8. doi: 10.1016/j.cortex.2018.09.014

118. Monda V, Villano I, Messina A, Valenzano A, Esposito T, Moscatelli F, et al. Exercise Modifies the Gut Microbiota With Positive Health
Effects. Oxid Med Cell Longev (2017) 2017:3831972. doi: 10.1155/2017/ 3831972

119. Pickard JM, Zeng MY, Caruso R, Nunez G. Gut Microbiota: Role in Pathogen Colonization, Immune Responses, and Inflammatory Disease. Immunol Rev (2017) 279(1):70-89. doi: 10.1111/imr.12567

120. Vogt NM, Kerby RL, Dill-McFarland KA, Harding SJ, Merluzzi AP, Johnson SC, et al. Gut Microbiome Alterations in Alzheimer's Disease. Sci Rep (2017) 7(1):13537. doi: 10.1038/s41598-017-13601-y

121. Yano JM, Yu K, Donaldson GP, Shastri GG, Ann P, Ma L, et al. Indigenous Bacteria From the Gut Microbiota Regulate Host Serotonin Biosynthesis. Cell (2015) 161(2):264-76. doi: 10.1016/j.cell.2015.02.047

122. Wipfli B, Landers D, Nagoshi C, Ringenbach S. An Examination of Serotonin and Psychological Variables in the Relationship Between Exercise and Mental Health. Scand J Med Sci Sports (2011) 21(3):474-81. doi: 10.1111/ j.1600-0838.2009.01049.x

123. Park SA, Son SY, Lee AY, Park HG, Lee WL, Lee CH. Metabolite Profiling Revealed That a Gardening Activity Program Improves Cognitive Ability Correlated With BDNF Levels and Serotonin Metabolism in the Elderly. Int J Environ Res Public Health (2020) 17(2):541. doi: 10.3390/ ijerph17020541

124. Lustgarten MS, Price LL, Fielding RA. Analytes and Metabolites Associated With Muscle Quality in Young, Healthy Adults. Med Sci Sports Exerc (2015) 47(8):1659-64. doi: 10.1249/MSS.0000000000000578

125. Lustgarten MS, Fielding RA. Metabolites Associated With Circulating Interleukin-6 in Older Adults. J Gerontol A Biol Sci Med Sci (2017) 72 (9):1277-83. doi: 10.1093/gerona/glw039

126. Smith PJ, Merwin RM. The Role of Exercise in Management of Mental Health Disorders: An Integrative Review. Annu Rev Med (2020) 72:45-62. doi: 10.1146/annurev-med-060619-022943

127. Klein AB, Williamson R, Santini MA, Clemmensen C, Ettrup A, Rios M, et al. Blood BDNF Concentrations Reflect Brain-Tissue BDNF Levels Across Species. Int J Neuropsychopharmacol (2011) 14(3):347-53. doi: 10.1017/ S1461145710000738

128. Pan W, Banks WA, Fasold MB, Bluth J, Kastin AJ. Transport of BrainDerived Neurotrophic Factor Across the Blood-Brain Barrier. Neuropharmacology (1998) 37(12):1553-61. doi: 10.1016/S0028-3908(98) 00141-5

129. Contrepois K, Wu S, Moneghetti KJ, Hornburg D, Ahadi S, Tsai MS, et al. Molecular Choreography of Acute Exercise. Cell (2020) 181(5):1112-30. doi: 10.1016/j.cell.2020.04.043

130. Gonulalan EM, Nemutlu E, Bayazeid O, Koçak E, Yalçın FN, Demirezer LO. Metabolomics and Proteomics Profiles of Some Medicinal Plants and Correlation With BDNF Activity. Phytomedicine (2020) 74:152920. doi: 10.1016/j.phymed.2019.152920

131. Voss MW, Nagamatsu LS, Liu-Ambrose T, Kramer AF. Exercise, Brain, and Cognition Across the Life Span. J Appl Physiol (1985) (2011) 111(5):1505-13. doi: 10.1152/japplphysiol.00210.2011

132. Erickson KI, Hillman C, Stillman CM, Ballard RM, Bloodgood B, Conroy DE, et al. Physical Activity, Cognition, and Brain Outcomes: A Review of the 2018 Physical Activity Guidelines. Med Sci Sports Exerc (2019) 51(6):124251. doi: $10.1249 /$ mss.0000000000001936

Conflict of Interest: The authors declare that the research was conducted in the absence of any commercial or financial relationships that could be construed as a potential conflict of interest.

Copyright $\odot 2021$ Gaitán, Moon, Stremlau, Dubal, Cook, Okonkwo and van Praag. This is an open-access article distributed under the terms of the Creative Commons Attribution License (CC BY). The use, distribution or reproduction in other forums is permitted, provided the original author(s) and the copyright owner(s) are credited and that the original publication in this journal is cited, in accordance with accepted academic practice. No use, distribution or reproduction is permitted which does not comply with these terms. 